\title{
Nox4 Mediates RANKL-induced ER-Phagy and Osteoclastogenesis via Activating ROS/PERK/elF- 2a/ATF4 Pathway
}

\section{Jing Sun}

Xinqiao Hospital of Army Medical University

wugui chen

Xinqiao Hospital

Songtao Li

Xinqiao Hospital

Sizhen Yang

Xinqiao Hospital

Ying Zhang

Xinqiao Hospital

$\mathrm{Xu} \mathrm{Hu}$

Xinqiao Hospital

Hao Qiu

Xinqiao Hospital

Shangcheng $\mathrm{Xu}$

The Sixth People's Hospital of Chongqing

Tongwei Chu ( $\sim$ chtw@sina.com )

Xinqiao Hospital https://orcid.org/0000-0003-0309-7082

\section{Research}

Keywords: Receptor activator of nuclear factor-KB ligand (RANKL), Osteoclastogenesis, Endoplasmic reticulum autophagy (ER-phagy), Nox4, Reactive oxygen species (ROS), PERK/elF-2a/ATF4 pathway

Posted Date: June 11th, 2020

DOI: https://doi.org/10.21203/rs.3.rs-34288/v1

License: (c) (i) This work is licensed under a Creative Commons Attribution 4.0 International License.

Read Full License 


\section{Abstract}

Background: Receptor activator of nuclear factor-KB ligand (RANKL) has been found to induce osteoclastogenesis and bone resorption. However, the underlying molecular mechanisms remain unclear.

Methods: Osteoclastogenesis was evaluated by number of TRAP-positive multinuclear ( $\geq 3$ ) osteoclasts, bone resorption pits and expression levels of related genes. Autophagy activity were evaluated by LC3II/LC3-I ratio, number of autophagic vacuoles and adenovirus-mRFP-GFP-tagged LC3 reporting system; Inhibitor chloroquine (CQ) was used to verified the role of autophagy in RANKL-induced osteoclastogenesis; Via downregulating Nox4 with inhibitor (DPI) and retrovirus-conveyed shRNA, we further explored the importance of Nox4 in RANKL-induced autophagy and osteoclastogenesis, as well as the regulatory effects of Nox4 on nonmitochondrial reactive oxygen species (ROS) and PERK/elF2a/ATF4 pathway. Intracellular ROS scavenger (NAC), mitochondrial-targeted antioxidant (MitoTEMPO) and inhibitor of PERK (GSK2606414) were also employed to investigate the role of ROS and PERK/elF2a/ATF4 pathway in RANKL-induced autophagy and osteoclastogenesis.

Results: RANKL markedly increased autophagy, while CQ treatment caused reduction of RANKL-induced autophagy and osteoclastogenesis. Consistent with the increased autophagy, the protein levels of Nox4 were significantly increased, and Nox4 was selectively localized within the endoplasmic reticulum (ER) after RANKL stimulation. DPI and shRNA efficiently decreased the protein level and (or) activity of Nox4 in the ER and inhibited RANKL-induced autophagy and osteoclastogenesis. Mechanistically, we found that Nox4 regulates RANKL-induced autophagy activation and osteoclastogenesis by stimulating the production of nonmitochondrial ROS. Additionally, Nox4-derived nonmitochondrial ROS dramatically activate PERK/elF-2a/ATF4, which is a critical unfolded protein response (UPR)-related signaling pathway during ER stress. Blocking the activation of the PERK/elF-2a/ATF4 signaling pathway either by Nox4 shRNA, ROS antioxidant or PERK inhibitor (GSK2606414) treatment significantly inhibited endoplasmic reticulum autophagy (ER-phagy) during RANKL-induced osteoclastogenesis.

Conclusions: Our findings provide new insights into the processes of RANKL-induced osteoclastogenesis and will help the development of new therapeutic strategies for osteoclastogenesis-related diseases.

\section{Introduction}

Throughout life, bone homeostasis is maintained through elaborate remodeling via coordinated bone formation and bone resorption [1]. Osteoclasts (OCs) are the principal cells responsible for bone resorption [2]. OCs, characterized as tartrate-resistant acid phosphatase (TRAP)-positive, are derived from the hematopoietic monocyte/macrophage lineage, and fuse to form multinucleated cells by an orchestrated process $[3,4]$. The excessive differentiation of osteoblasts is the pathological basis of a variety of osteolytic diseases, such as postmenopausal osteoporosis, Paget disease of bone (PDB) and inflammatory arthritis $[5,6]$. Therefore, there is no doubt that identifying pharmacological inhibitors targeting osteoclasts differentiation will help the development of new prophylactic and therapeutic 
strategies for osteolytic bone lesions [7]. The differentiation and maturation of osteoclasts is a complicated process that is regulated by various cytokines [8]. RANKL, a member of the tumor necrosis factor (TNF) superfamily [9], has been demonstrated to interact with the RANK receptor expressed on osteoclast precursors to activate multiple osteoclastogenesis-related signaling pathways (NF-kB, Src, MAPK, etc.), releasing nuclear transcription factors (NFATc1, AP-1, etc.) and regulating the expression of osteoclastogenesis-related genes, which induce the differentiation and maturation of osteoclasts in the bone microenvironment $[10,11]$. Moreover, denosumab, a monoclonal antibody with activity against RANKL, has been demonstrated to be effective in the prevention and treatment of osteolytic disease [12]. However, the detailed mechanisms of osteoclastogenesis induced by RANKL remain unclear.

Autophagy, an evolutionarily conserved and dynamic catabolic process that delivers cellular components to the lysosome for degradation [13], is strongly associated with the development and differentiation of multiple cell types, including lymphocytes, adipocytes, chondrocytes, neurons, and erythrocytes [14-19]. Previous studies have revealed that the upregulation of autophagy contributes to osteoclastogenesis in response to hypoxic conditions, glucocorticoid treatment and microgravity in vitro [20-22]. Moreover, several key autophagy-regulated proteins such as LC3 and Atg5 have been shown to participate in osteoclast bone resorption by directing lysosomal content secretion into the extracellular space [23]. However, it is still unclear whether autophagy is involved in RANKL-induced osteoclastogenesis.

Here, we found for the first time that the increase in Nox4 protein levels in the ER contributes to the activation of autophagy during RANKL-induced osteoclastogenesis. Nonmitochondrial ROS, but not mitochondrial ROS, are closely associated with the critical roles of Nox4 in regulating RANKL-induced autophagy activation and osteoclastogenesis. Inhibiting Nox4 expression via shRNA or blocking ROS inhibited the activation of the PERK/elF-2 $\alpha$ /ATF4 signaling pathway and then inhibited autophagy and ER-phagy during RANKL-induced osteoclastogenesis. Our findings provide new insight into the processes of RANKL-induced osteoclastogenesis and will facilitate the development of new therapeutic strategies for osteoclastogenesis-related diseases.

\section{Materials And Methods}

\subsection{Reagents and antibodies}

Recombinant murine soluble RANKL (sRANKL) was purchased from Peprotech (London, UK). Chloroquine (CQ) phosphate was obtained from Selleck (Shanghai, China). ML171 was acquired from Millipore Corporation (Temecula, CA, USA). Diphenyleneiodonium chloride (DPI) and 5-0-methyl quercetin were purchased from Santa Cruz Biotechnology (Dallas, TX, USA). N-acetyl-L-cysteine (NAC) and MitoTEMPO were purchased from Sigma-Aldrich (St. Louis, MO, USA). GSK2795039 and GSK2606414 were provided by MCE (Shanghai, China).

Both the mouse Nox4 short hairpin RNA (shRNA)-containing retrovirus and the corresponding empty vector were designed and synthesized by HanBio (Shanghai, China). The mRFP-GFP-LC3-containing adenovirus was provided by HanBio. Primary antibodies against Nox3, Nox4, ATF6, ATF4, XBP1, GAPDH, 
ERp57/ERp60 and VDAC1/Porin were obtained from Proteintech (Wuhan, Hubei, China). Primary antibodies against LC3A/B, PERK (C33E10), phospho-PERK (Thr980; 16F8), elF2a (D7D3) XP ${ }^{\circledR}$ and phospho-elF2a (Ser51; D9G8) XP ${ }^{\circledR}$ were purchased from Cell Signaling Technology (Danvers, Massachusetts, USA). Primary antibodies against Nox1 and Nox2/gp91phox were obtained from Abcam (Cambridge, UK).

\subsection{Cell culture}

The RAW264.7 mouse monocyte/macrophage cell line was purchased from the Cell Culture Center of the Chinese Academy of Sciences (Shanghai, China) and cultured in Dulbecco's modified Eagle's medium (DMEM) (Gibco, Grand Island, NY, USA) supplemented with 10\% fetal bovine serum (FBS) (Gibco). Containing $100 \mathrm{U} / \mathrm{mL}$ penicillin and $100 \mathrm{mg} / \mathrm{mL}$ streptomycin (Gibco). The cells were incubated in a humidified atmosphere with $95 \%$ air and $5 \% \mathrm{CO}_{2}$ at $37^{\circ} \mathrm{C}$. To induce osteoclast differentiation, RAW264.7 cells were stimulated with $100 \mathrm{ng} / \mathrm{mL}$ RANKL and further cultured for the indicated times.

\subsection{Retrovirus-mediated stable knockdown of Nox4}

RAW264.7 cells were plated and cultured in 35-mm dishes. When the confluence reached $50 \%$, the cells were transfected with retrovirus encoding Nox4 shRNAs or scrambled shRNA at a multiplicity of infection (MOI) of 100 for $24 \mathrm{~h}$ according to the manufacturer's instructions. The nucleotide sequences were as follows: sh-Nox4-1, 5'-GCAGGAGAACCAGGAGATTGT-3'; sh-Nox4-2, 5'-GCATGGTGGTGGTGCTATT CC-3'; sh-Nox4-3, 5'-GGTATACTCATAACCTCTTCT-3'; and sh-NC, 5'-TTCTCCGAA CGTGTCACGT-3'. RAW264.7 cells with stable knockdown of Nox4 expression were screened by the addition of $2 \mu \mathrm{g} / \mathrm{mL}$ puromycin to the culture medium for $48 \mathrm{~h}$. Then, the stable cells were digested with $0.25 \%$ trypsin and seeded on 35 $\mathrm{mm}$ dishes at a density of $8 \times 10^{4}$ cells/dish and incubated overnight for attachment. The next day, adherent cells were treated with or without RANKL $(100 \mathrm{ng} / \mathrm{mL})$ for 3 days. The knockdown efficiency of the three Nox4 shRNAs was measured using western blotting, and the most effective was selected for use in subsequent experiments.

\subsection{Osteoclast differentiation assay}

Osteoclast formation was measured by quantifying cells positively stained with TRAP. Briefly, RAW264.7 cells were incubated at a density of $1 \times 10^{4}$ cells/well in 24-well plates overnight. After stimulation with RANKL (100 ng/mL) and various concentrations of different pharmacological reagents for 6 days, the cells were fixed with $4 \%$ paraformaldehyde for $30 \mathrm{~min}$ at room temperature and then stained by using a Tartrate Resistant Acid Phosphatase Assay Kit (Sigma-Aldrich) according to the manufacturer's instructions. TRAP-positive and multinucleated cells containing three or more nuclei were considered osteoclasts. For each well, the osteoclasts were observed under a light microscope (Leica, Wetzlar, Germany).

\subsection{Osteoclast bone resorption pit formation assay}


To confirm the bone resorption ability of differentiated osteoclasts, RAW264.7 cells were seeded at a density of $2 \times 10^{4}$ cells/well overnight in 24-well Osteo Assay Surface plates (Corning, New York, NY, USA) coated with hydroxyapatite matrix. Then, the cells were incubated with RANKL $(100 \mathrm{ng} / \mathrm{mL})$ or in the presence of various concentrations of different pharmacological reagents. The medium was replaced every 3 days. After 7 days of culture, the cells were removed using a 10\% sodium hypochlorite solution, and the wells were stained with $1 \%$ toluidine blue. The plate was washed twice with distilled water and air dried at room temperature. The areas of bone resorption pits in each well were determined using a light microscope (Leica).

\subsection{Quantitative real-time PCR (qRT-PCR)}

Total cellular RNA was extracted using RNAiso plus reagent (Takara, Kyoto, Japan) according to the manufacturer's instructions. Subsequently, the total RNA concentration was determined with a NanoDrop 2.0 spectrophotometer (Thermo Fisher Scientific, Pittsburgh, PA, USA), and the RNA was reverse transcribed to $c D N A$ using a Prime Script ${ }^{\mathrm{TM}}$ RT reagent kit with gDNA Eraser (Takara) according to the manufacturer's instructions. Subsequently, qRT-PCR assays were performed by using a SYBR Premix Ex $\mathrm{Taq}^{\mathrm{TM}}$ II (2x) kit (Takara) according to the manufacturer's instructions and run on an ABI 7500 Real-Time PCR Detection System (Foster City, CA, USA). The reactions were performed using the following parameters: $95^{\circ} \mathrm{C}$ for $30 \mathrm{~s}$ followed by 40 cycles of $95^{\circ} \mathrm{C}$ for $5 \mathrm{~s}$ and $60^{\circ} \mathrm{C}$ for $30 \mathrm{~s}$. The primer nucleotide sequences used for PCR are listed in Table 1. All primer sets for mRNA amplification were purchased from Sangon Biotech (Shanghai) Co., Ltd. (Shanghai, China). The relative expression levels of the target gene were normalized with respect to the levels of $\beta$-actin expression and calculated using the $2^{-} \triangle \triangle \mathrm{CT}$ method.

\section{Table 1. Primer sequences used for real-time quantitative PCR}

\begin{tabular}{|c|c|c|}
\hline Gene & Sequence (5' to $\left.3^{\prime}\right)$ & Amplicon size (bp) \\
\hline \multirow[t]{2}{*}{ TRAP } & Forward: ACTTGCGACCATTGTTAGCCACAT & \multirow[t]{2}{*}{91} \\
\hline & Reverse: ACACCGTTCTCGTCCTGAAGATACT & \\
\hline \multirow[t]{2}{*}{ MMP-9 } & Forward: CCAGTATCTGTATGGTCGTGGCTCTA & \multirow[t]{2}{*}{82} \\
\hline & Reverse: AGGTGCTGTCGGCTGTGGTT & \\
\hline \multirow[t]{2}{*}{ Cath K } & Forward: AGGATATGCTCTCTTGGCTCGGAAT & \multirow[t]{2}{*}{91} \\
\hline & Reverse: GCTGGCTGGCTGGAATCACATC & \\
\hline \multirow[t]{2}{*}{$\beta$-actin } & Forward: TCACTATTGGCAACGAGCGGTTC & \multirow[t]{2}{*}{153} \\
\hline & Reverse: GCACTGTGTTGGCATAGAGGTCTT & \\
\hline
\end{tabular}

\subsection{Transmission electron microscopy (TEM)}


RAW264.7 cells were cultured with the indicated treatments for 3 days. Then, the cells were digested with $0.25 \%$ trypsin, centrifuged $(2000 \mathrm{rpm})$ for $10 \mathrm{~min}$ and fixed with $2.5 \%$ glutaraldehyde overnight at $4{ }^{\circ} \mathrm{C}$. Subsequently, the cells were postfixed with $1 \%$ osmium tetroxide for $1.5 \mathrm{~h}$, washed and stained in $3 \%$ aqueous uranyl acetate for $1 \mathrm{~h}$. Thereafter, the samples were washed again, dehydrated with a graded series of increasing ethanol concentrations to $100 \%$ and embedded in Epon-Araldite resin. Subsequently, the ultrathin sections were cut using a Reichert ultramicrotome (Reichert, New York, NY, USA) and counterstained with $0.3 \%$ lead citrate. Then, the ultrastructure of autophagic vacuoles (autophagosomes and autolysosomes) was observed under a Philips EM420 transmission electron microscope (Philips, UK), and images were captured.

\subsection{Autophagic flux assessment}

After growth to $50 \%$ confluence in $35-\mathrm{mm}$ dishes, the cells were transfected with adenovirus expressing mRFP-GFP-LC3 (HanBio) for $24 \mathrm{~h}$ using an MOI of 1000, according to the manufacturer's instructions. Then, the cell growth medium was replaced with fresh complete medium for another $24 \mathrm{~h}$. Afterward, the transfected cells were digested with $0.25 \%$ trypsin and seeded on confocal Petri dishes (NEST, Wuxi, Jiangsu, China) at a density of $5 \times 10^{4}$ cells/dish and incubated overnight for attachment. Thereafter, adherent cells were treated with the various indicated treatments for 3 days. The treated cells were washed with phosphate buffer saline (PBS) and viewed with a laser scanning confocal microscope (Leica). GFP loses its fluorescence in acidic lysosomal conditions, whereas mRFP does not. Therefore, yellow (merged GFP signal and RFP signal) puncta represent early autophagosomes, whereas puncta detectable only as red (RFP signal alone) indicate late autolysosomes that are formed by autophagosome fusion with lysosomes. Autophagic flux was ultimately assessed by quantifying the mRFP and GFP puncta per cell. The number of GFP and mRFP puncta was determined by manually counting 30 cells randomly in 5 fields per dish, and the average number of puncta per cell was calculated.

\subsection{ER-Tracker staining in living cells}

RAW264.7 cells $\left(5 \times 10^{4}\right)$ were plated on confocal Petri dishes (NEST) and allowed to attach overnight. Then, the cells were cultured with the indicated treatments for the indicated times. Next, ER-Tracker (Thermo Fisher Scientific) was added directly to the culture medium at $500 \mathrm{nM}$ and incubated with cells for $30 \mathrm{~min}$ in a $37^{\circ} \mathrm{C}$ humidified incubator containing $5 \% \mathrm{CO}_{2}$. Then, the cells were washed with PBS and immediately observed under a laser scanning confocal microscope (Leica).

\subsection{Immunofluorescence staining for Nox4 localization}

The colocalization of Nox4 with the ER was detected by double-labeling immunofluorescence. Briefly, RAW264.7 cells were seeded on confocal Petri dishes (NEST) at a density of $5 \times 10^{4}$ cells/dish overnight. Then, the cells were incubated with or without RANKL $(100 \mathrm{ng} / \mathrm{mL})$ for 3 days. Thereafter, the cells were stained with ER-Tracker (the detailed experimental procedure is described in step 2.9) and fixed in $4 \%$ paraformaldehyde for $20 \mathrm{~min}$ at $37^{\circ} \mathrm{C}$. Then, the cells were permeabilized with $0.1 \%$ Triton X-100 for 10 min and blocked with $10 \%$ normal goat serum for $1 \mathrm{~h}$ at $37^{\circ} \mathrm{C}$. Subsequently, the cells were incubated 
with a rabbit polyclonal anti-Nox4 antibody (1:25) in a humidified chamber at $4^{\circ} \mathrm{C}$ overnight. Then, the cells were washed and incubated with Alexa Fluor 488-labeled goat anti-rabbit IgG (1:500), (Beyotime Biotechnology, Nantong, Jiangsu, China) for $1.5 \mathrm{~h}$ at $37^{\circ} \mathrm{C}$ in the dark. DAPI Staining Solution (Beyotime Biotechnology) was used to counterstain the cell nuclei. Finally, the cells were observed using a laser scanning confocal microscope (Leica).

\subsection{Subcellular fractionation assay}

RAW264.7 cells were seeded in 6-cm dishes overnight. Then, the cells were cultured with the indicated treatments for the indicated times. Subsequently, the cells were digested with $0.25 \%$ trypsin and centrifuged (1000 rpm) for $5 \mathrm{~min}$. Mitochondria and the ER were extracted from cells using a Cell Mitochondria Isolation kit (Beyotime Biotechnology) and Endoplasmic Reticulum Isolation kit (BestBio Science, Shanghai, China) according to the manufacturer's instructions. The fractions of mitochondria and ER were lysed in RIPA buffer (Beyotime Biotechnology) that contained a protease and phosphatase inhibitor cocktail (Beyotime Biotechnology) for subsequent western blot analysis.

\subsection{Western blot analysis}

RAW264.7 cells were lysed in RIPA buffer (Beyotime Biotechnology) that contained a protease and phosphatase inhibitor cocktail (Beyotime Biotechnology). After centrifugation at $14000 \times \mathrm{g}$ for $5 \mathrm{~min}$ at $4^{\circ} \mathrm{C}$, the concentrations of protein were measured using a BCA protein assay kit (Beyotime Biotechnology). Subsequently, equal amounts of protein $(40 \mu \mathrm{g})$ were separated by $6 \%, 8 \%$ or $12 \% / 5 \%$ $(w / v)$ sodium dodecyl sulfate-polyacrylamide (SDS-PAGE) gel electrophoresis (Bio-Rad, Hercules, CA, USA) and transferred to polyvinylidene difluoride (PVDF) membranes. The membranes were incubated with anti-GAPDH, anti-Nox4, anti-LC3A/B, anti-ERp57/ERp60, anti-VDAC1/Porin, anti-Nox3, antiNox2/gp91phox, anti-Nox1, anti-PERK (C33E10), anti-phospho-PERK (Thr980) (16F8), anti-ATF6, antiATF4, anti-XBP1, anti-elF2a (D7D3) XP ${ }^{\circledR}$ and anti-phospho-elF2a (Ser51; D9G8) XP ${ }^{\circledR}$ antibodies separately overnight at $4{ }^{\circ} \mathrm{C}$. Then, the membranes were incubated with horseradish peroxidase (HRP)-conjugated goat anti-mouse/rabbit IgG ( $\mathrm{H}+\mathrm{L})$ secondary antibodies (1:5000) (ZSGB-BIO, Beijing, China) for $1 \mathrm{~h}$ at room temperature. Finally, the protein bands of interest on the membranes were visualized with a chemiluminescence substrate (ECL) kit (Millipore Corporation) using an Image Quant LAS4000 instrument (GE, UK). The band intensity was quantified by densitometric analysis using Image $\mathrm{J}$ software (National Institutes of Health, Bethesda, MD, USA).

\subsection{Determination of intracellular and ER ROS}

The intracellular production of ROS was detected by staining cells with a Reactive Oxygen Species Assay kit (Beyotime Biotechnology). Briefly, RAW264.7 cells $\left(5 \times 10^{4}\right)$ were seeded in confocal Petri dishes (NEST) overnight. Then, the adherent cells were cultured under conditions with various treatments for the indicated times. Subsequently, the cells were stained with ER-Tracker (the detailed experimental procedure is described in step 2.9) and washed with PBS. Then, 2',7'-dichlorofluorescein diacetate (DCFH-DA), which was added directly to serum-free medium, was diluted to a final concentration of $10 \mu \mathrm{M}$ and incubated 
with cells for $30 \mathrm{~min}$ at $37^{\circ} \mathrm{C}$ in a humidified incubator containing $5 \% \mathrm{CO}_{2}$. DCFH-DA diffuses into cells and is deacetylated by cellular esterases to nonfluorescent (DCFH), which can be oxidized by ROS to produce highly fluorescent 2',7'-dichlorofluorescein (DCF). The green fluorescence intensity is proportional to the levels of ROS within a cell. The cells were then washed three times with PBS, and the fluorescence intensity was observed using a laser scanning confocal microscope.

\subsection{Measurement of mitochondrial ROS}

The levels of mitochondrial ROS were measured by staining cells with MitoSOX ${ }^{\mathrm{TM}}$ Red Mitochondrial Superoxide Indicator (Thermo Fisher Scientific). Briefly, RAW264.7 cells were seeded at a density of $3 \times$ $10^{3}$ cells/well on 96-well plates and allowed to attach overnight. Then, the cells were cultured with various treatments for the indicated times. Subsequently, the cells were incubated with MitoSOX at a final concentration of $5 \mu \mathrm{M}$ for $15 \mathrm{~min}$ at $37^{\circ} \mathrm{C}$ in a humidified incubator containing $5 \% \mathrm{CO}_{2}$. The red fluorescence intensity is proportional to the levels of mitochondrial ROS within the cell. Then, the cells were washed three times with PBS, and the fluorescence intensity was immediately measured with a Varioskan Flash Spectral Scanning Multimode Reader (Thermo Fisher Scientific).

\subsection{Statistical analysis}

The data are expressed as the mean \pm SD of three independent experiments. The difference in means between 2 groups was compared using Student's t-test. The data for multiple groups were analyzed by one-way analysis of variance (ANOVA) with subsequent post hoc multiple comparisons by Dunnett's test. Statistical analyses were performed with the use of GraphPad Prism 5.00 (GraphPad Software, San Diego, CA, USA). Differences with $P$ values $<0.05$ were considered statistically significant.

\section{Results}

\subsection{RANKL induces osteoclastogenesis and bone resorption via autophagy}

Consistent with that reported in previous studies, we found that RANKL enhanced the proportion of fused multinuclear cells (Supplemental Fig. 1A) and the expression levels of osteoclastogenesis-related genes (TRAP, Cath K and MMP-9; Supplemental Fig. 1B), inducing the formation of TRAP-positive multinuclear ( $\geq 3$ ) osteoclasts and bone resorption pits (Supplemental Fig. 1C) in RAW264.7 cells. These results indicate that RANKL induced the differentiation and subsequent bone resorption activity of osteoclasts in vitro, which is consistent with previous findings [24].

Autophagy has been demonstrated to play critical roles in enhanced osteoclastogenesis under many conditions, such as hypoxia, oxidative stress and microgravity $[22,25,26]$. First, we assayed the level of autophagy after RANKL treatment. Western blot analysis showed that the LC3-II/LC3-I ratio was significantly upregulated from day 3 in a time-dependent manner during RANKL-induced osteoclastogenesis (Fig. 1A). The TEM showed that the number of autophagic vacuoles was dramatically increased after 3 days of treatment with RANKL (Fig. 1C). Then, the autophagic flux activity was further 
determined by using the adenovirus-mRFP-GFP-tagged LC3 system. The data showed that the number of yellow and red puncta in merged images was significantly increased after 3 days of RANKL-induced differentiation, indicating the activation of both autophagosome formation and lysosomal degradation in the RANKL-treated group (Fig. 1D). Collectively, these observations indicate that autophagy is activated during RANKL-induced osteoclastogenesis. Second, using a pharmacological inhibitor of autophagy (CQ), we explored whether autophagy is essential in RANKL-induced osteoclastogenesis. The results showed that CQ treatment markedly increased the LC3-II/LC3-I ratio (Fig. 1B) and inhibited autolysosomal degradation (Fig. 1C, D). More importantly, we found that CQ treatment suppressed the RANKL-induced upregulation of osteoclastogenesis-related genes (TRAP, Cath K and MMP-9; Fig. 1E), reduced the number of TRAP-positive multinuclear $(\geq 3$ ) osteoclasts and reduced the area of the bone resorption pits (Fig. $1 \mathrm{~F}$ ). Taken together, the above data reveal that RANKL induces osteoclastogenesis and bone resorption through autophagy.

\subsection{Downregulation of Nox4 suppresses RANKL-induced autophagy and osteoclastogenesis}

The above experiments showed that RANKL induced osteoclastogenesis and bone resorption through autophagy; we next uncovered the molecular mechanism of RANKL-induced autophagy. It is well known that Nox family proteins promote the activation of autophagy in many cell types by generating ROS [27]. Recent evidence indicated that RANKL increases the generation of intracellular ROS by promoting the expression and activity of intracellular Nox family proteins during osteoclastogenesis [28-30]. Therefore, we explored whether Nox family proteins are involved in RANKL-induced autophagy.

Western blot analysis showed that RANKL time-dependently upregulated the levels of Nox1 and Nox4 proteins and decreased the levels of Nox2 protein but had no significant influence on the levels of Nox3 protein (Fig. 2A). To further investigate whether Nox family proteins are involved in RANKL-induced autophagy and osteoclastogenesis, the Nox pharmacological inhibitor DPI was utilized in the presence of RANKL. The data showed that DPI treatment obviously downregulated the RANKL-induced increase in the LC3-II/LC3-I ratio (Fig. 2B). To further investigate which Nox isoforms are involved in RANKL-induced autophagy and osteoclastogenesis, pharmacological inhibitors targeting specific Nox isoforms were used. The data showed that the inhibition of Nox1 or Nox4 (not Nox2) separately by ML171 and 5-Omethyl quercetin significantly inhibited the RANKL-induced increase in the LC3-II/LC3-I ratio and osteoclastogenesis-related gene (TRAP, Cath K and MMP-9) expression and reduced the number of TRAPpositive multinuclear $(\geq 3$ ) osteoclasts and the area of the bone resorption pits (Fig. 2C-F). Importantly, the inhibitory effect of 5-0-methyl quercetin on RANKL-induced autophagy, osteoclastogenesis and bone resorption was significantly greater than that of ML171. Therefore, compared with Nox1, Nox4 may play a leading role in autophagy activation induced by RANKL. We selected Nox4 for the subsequent experiments.

To further determine the functional significance of Nox4 in RANKL-induced autophagy and osteoclastogenesis, retroviruses encoding three different Nox4 shRNAs or scrambled shRNA were utilized. The results showed that the expression levels of Nox4 were significantly decreased after the transfection 
of sh-Nox4-1, sh-Nox4-2 and sh-Nox4-3 (Supplemental Fig. 2A). Importantly, sh-Nox4-2 had the greatest Nox4 silencing effect. Therefore, sh-Nox4-2 was selected for the subsequent experiments. The knockdown of Nox4 markedly inhibited the RANKL-induced increase in the LC3-II/LC3-I ratio, autophagic flux activity, and expression of osteoclastogenesis-related genes (TRAP, Cath K and MMP-9) and reduced the number of TRAP-positive multinuclear $(\geq 3)$ osteoclasts and bone resorption pit area (Supplemental Fig. 2B-E). Collectively, the above results indicate that knockdown of Nox4 suppresses RANKL-induced autophagy and osteoclastogenesis.

\subsection{RANKL specifically upregulates the level of Nox4 protein in the ER}

Recent studies have indicated that Nox4 is localized on intracellular membranes, mainly in mitochondria and the ER [31]. To determine in which subcellular compartment Nox4 protein is located during RANKLinduced osteoclastogenesis, we investigated alterations in Nox4 protein expression levels in the ER and mitochondria in RAW264.7 cells cultured under RANKL induction conditions. Western blot analysis showed that RANKL treatment markedly increased the protein level of Nox4 in the ER (not in the mitochondria) of RAW264.7 cells (Fig. 3A-B). As shown in Fig. 4C, Nox4 shRNA treatment significantly downregulated the RANKL-induced increase in the Nox4 protein level in the ER of RAW264.7 cells (Fig. 3C). To further ascertain the ER localization of Nox4 protein induced by RANKL, an immunofluorescence staining assay was utilized. The results showed that RANKL treatment significantly enhanced the localization of Nox4 in the ER, which was markedly suppressed by Nox4 silencing (Fig. 3D). Collectively, the above data reveal that RANKL specifically upregulates the level of Nox4 protein in the ER. This finding is consistent with the fact that membrane proteins are mainly synthesized in the ER and with the evidence that the N-terminal portion of Nox4 contains multiple ER-specific signal sequences [32, 33].

\subsection{Nox4 promotes RANKL-induced autophagy activation and osteoclastogenesis by generating nonmitochondrial ROS}

It has been reported that Nox family proteins can promote the production of intracellular ROS [34]. Therefore, the level of ROS was assayed by a fluorescence staining assay. As shown in Fig. 4A, RANKL treatment markedly enhanced the level of intracellular ROS and ER ROS in RAW264.7 cells, which was reduced by Nox4 silencing. As shown in Fig. 4C, the level of mitochondrial ROS was increased in RAW264.7 cells during RANKL-induced osteoclastogenesis. As ROS have been reported to play an important role in autophagy regulation [35], we explored whether ROS are involved in RANKL-induced autophagy and osteoclastogenesis. The data showed that intracellular ROS scavenger (NAC) treatment significantly inhibited the RANKL-induced accumulation of intracellular ROS and ER ROS (Fig. 4B). Mitochondrial-targeted antioxidant (MitoTEMPO) treatment significantly inhibited RANKL-induced mitochondrial ROS accumulation (Fig. 4C). Importantly, MitoTEMPO treatment did not affect the RANKLinduced increase in the LC3-II/LC3-I ratio (Fig. 4D), whereas NAC treatment obviously reduced the RANKLinduced increase in the LC3-II/LC3-I ratio (Fig. 4D) and the number of yellow and red puncta in merged images, which implies an impairment in autophagic flux activity (Fig. 4E). Additionally, NAC treatment also significantly downregulated the RANKL-induced upregulation of the expression of

Page $10 / 30$ 
osteoclastogenesis-related genes (TRAP, Cath K and MMP-9; Fig. 4F) and reduced the number of TRAPpositive multinuclear ( $\geq 3$ ) osteoclasts and bone resorption pit area (Fig. 4G). In summary, these data indicate that Nox4 promotes RANKL-induced autophagy activation and osteoclastogenesis by generating nonmitochondrial ROS. Furthermore, we also found that the majority of Nox4-derived ROS colocalize with ER-Tracker (Fig. 4A). These results suggest that Nox4 may promote the activation of autophagy via the generation of ER-derived ROS during RANKL-induced osteoclastogenesis.

\subsection{Nox4-derived ROS promotes autophagy via the PERK/elF-2a/ATF4 pathway}

Previous studies have demonstrated that UPR-related signaling pathways (ATF6, PERK/elF-2a/ATF4 and IRE-1 a/XBP-1) are involved in ROS-induced autophagy [36, 37]. Therefore, we further explored whether UPR-related signaling pathways mediate ROS-induced autophagy during RANKL-induced osteoclastogenesis. Western blot analysis showed that RANKL treatment time-dependently increased the phosphorylation of PERK (Thr980) and elF-2a (Ser51) and upregulated the expression levels of ATF4 and XBP-1S in RAW264.7 cells but had little influence on the level of ATF6 (Fig. 5A). Moreover, knockdown of Nox4 significantly repressed the RANKL-induced phosphorylation of PERK (Thr980) and elF-2a (Ser51) and the upregulation of ATF4 expression but had little influence on XBP-1S (Fig. 5B). Furthermore, NAC treatment significantly inhibited the RANKL-induced activation of the PERK/elF-2a/ATF4 pathway (Fig. 5C). These results reveal that RANKL activates the PERK/elF-2a/ATF4 pathway by elevating Nox4mediated ROS production in RAW264.7 cells. To further confirm the involvement of the PERK/elF$2 a / A T F 4$ pathway in the activation of autophagy, osteoclastogenesis and bone resorption, a pharmacological inhibitor of PERK (GSK2606414) was utilized. The data revealed that GSK2606414 treatment dose-dependently reduced the RANKL-induced phosphorylation of elF-2a (Ser51) and the upregulation of ATF4 expression in RAW264.7 cells (Fig. 5D). More importantly, GSK2606414 treatment obviously inhibited the RANKL-induced increase in the LC3-II/LC3-I ratio (Fig. 5E) and the number of yellow and red puncta in merged images, which implies that autophagic flux is impaired (Fig. 5F). Additionally, GSK2606414 treatment also significantly abrogated the RANKL-induced upregulation of osteoclastogenesis-related genes (TRAP, Cath K and MMP-9; Fig. 5G) and reduced the number of TRAPpositive multinuclear $(\geq 3$ ) osteoclasts and bone resorption pit area (Fig. $5 \mathrm{H}$ ). These results indicate that Nox4-derived ROS promote autophagy via the PERK/elF-2a/ATF4 pathway during RANKL-induced osteoclastogenesis.

\subsection{RANKL promotes ER-phagy via activating Nox4/ROS/PERK/elF-2a/ATF4 pathway}

Recent evidence has indicated that the activation of the UPR can selectively induce ER-phagy [38]. Therefore, we further validated whether UPR-related signaling pathways (PERK/elF-2a/ATF4) induce ERphagy during RANKL-induced osteoclastogenesis. The results showed that RANKL treatment markedly increased the ratio of LC3-II/LC3-I in the ER and the number of GFP-LC3 puncta colocalized with the ER, which was reduced by Nox4 silencing (Fig. 6A-C). These observations indicate that RANKL promotes ERphagy by upregulating the protein level of Nox4 in RAW264.7 cells. Moreover, we found that NAC and PERK inhibitor (GSK2606414) treatment can separately reduce the RANKL-induced increase in the LC3-

Page $11 / 30$ 
II/LC3-I ratio in the ER and number of GFP-LC3 puncta colocalized with the ER (Fig. 6D-G). Collectively, these results indicate that RANKL promotes ER-phagy by activating the Nox4/ROS/PERK/elF-2a/ATF4 pathway.

\section{Discussion}

Autophagy, an evolutionarily conserved and dynamic catabolic process, plays a critical role in maintaining bone homeostasis [39]. However, it is still unclear whether autophagy is involved in RANKLinduced osteoclastogenesis. In the present study, we identified a novel mechanism of autophagy regulation during RANKL-induced osteoclastogenesis. Specifically, ER-resident Nox4 promotes RANKLinduced autophagy activation and osteoclastogenesis by stimulating an increase in nonmitochondrial ROS. Nox4 shRNA or ROS antioxidant treatment inhibited the activation of the PERK/elF-2a/ATF4 signaling pathway and then suppressed autophagy and ER-phagy during RANKL-induced osteoclastogenesis. Our results provide new insight into the molecular mechanisms of RANKL-induced osteoclastogenesis and will help the development of new therapeutic strategies for osteoclastogenesisrelated diseases.

Nox, which is widely distributed in various tissues and organs, is the key enzyme of redox signaling [40]. The Nox family is composed of five different isoforms (Nox1, Nox2, Nox3, Nox4, Nox5) of a kind of transmembrane protein [41]. The protein levels of Nox1 and Nox4 are increased, the protein level of Nox2 is decreased, and the protein level of Nox3 remains unchanged during RANKL-induced osteoclastogenesis [42]. Compared with the other isoforms, Nox1 plays more prominent roles in stimulating RANKL-induced osteoclastogenesis [28]. In this study, we found that RANKL treatment caused similar Nox protein expression patterns. However, we found that Nox4 plays a more critical role in regulating RANKL-induced autophagy activation than other Nox isoforms, including Nox1. This specific role of Nox4 in regulating autophagy may be dependent upon its intracellular localization. In contrast to Nox1, which is mainly located on the plasma membrane, Nox4 is localized to intracellular membranes, particularly in the ER and mitochondria [31].

Nox4 is composed of conserved transmembrane domains, FAD- and NADPH-binding domains in the Cterminal region, and two heme groups $[43,44]$. ER-localized Nox4 has been found to promote the proliferation, migration, differentiation and survival of cells $[45,46]$. The activity of ER-localized Nox4 in the regulation of cellular processes may be dependent upon its ability to produce $\mathrm{H}_{2} \mathrm{O}_{2}$, which can be a stable and diffusible signaling molecule, through its E-loop portion [47]. Here, we observed for the first time that the level of ER-localized (not mitochondria-localized) Nox4 was dramatically increased during RANKL-induced osteoclastogenesis. This result may be explained by the fact that Nox4 is a membranebound protein and, therefore, is mainly translated in the ER through a cotranslational translocation mechanism [32]. However, it is very likely that the preferential cellular localization of Nox4 is stimulus dependent. Recent evidence suggests that alternative splicing of Nox4 mRNA may drive Nox4 synthesis in different subcellular compartments [48]. It is reasonable to speculate that posttranslational 
modifications of nascent Nox4 protein or other unknown mechanisms may be involved in this selective activation of Nox4 in the ER of RAW264.7 cells cultured under RANKL induction conditions [49].

Nox proteins are considered the most important source of ROS from different parts of the cell, including mitochondria [50], the ER [27] and the cytomembrane [26]. Increased intracellular ROS accumulation induces autophagy in various cell types [51,52]. Mitochondria, as the sites of oxidative respiration in cells, are the main production sites of intracellular ROS [53]. Previously, we and others have found that RANKL treatment increases mitochondrial ROS in osteoclast precursors [54]; however, mitochondrialtargeted antioxidants do not block RANKL-induced autophagy activation. These results indicate that Nox4 promotes the activation of autophagy by generating nonmitochondrial ROS during RANKL-induced osteoclastogenesis. Furthermore, we also found that the majority of Nox4-derived ROS were colocalized with ER-Tracker. Inhibiting the increase in Nox4 protein levels efficiently reduced ROS, reversed the activation of the PERK/elF-2a/ATF4 pathway and suppressed RANKL-induced autophagy and osteoclastogenesis. These results suggest that Nox4 may promote the activation of autophagy by generating ER-derived ROS during RANKL-induced osteoclastogenesis. This finding is supported by the findings of a recent study in which an increase in Nox4-dependent ROS accumulation in the ER of cardiomyocytes was found to promote the activation of autophagy and survival during energy deprivation [27].

ER-phagy, a selective form of autophagy in which portions of the ER are sequestered within autophagosomes and transported to the lysosomes for degradation [55], is considered to play an important role in the ER quality control (ERQC) system by removing excess or damaged ER components [56]. Previous studies have shown that under ER stress conditions, ER-phagy is required for ER turnover and cell survival $[57,58]$. Activation of the UPR can selectively induce ER-phagy to promote recovery after ER stress and maintain ER homeostasis [59]. However, there are a variety of ER-phagy regulatory mechanisms under different stimulation conditions, suggesting the complexity of ER-phagy. In the present study, we found that the Nox4-dependent accumulation of ROS promotes ER-phagy by activating the UPR-related signaling pathway (PERK/elF-2a/ATF4) during RANKL-induced osteoclastogenesis. To the best of our knowledge, this is the first study to reveal the novel role of the PERK/elF-2a/ATF4 pathway in regulating ER-phagy and enables us to explore novel molecular mechanisms of ER-phagy.

In conclusion, we identified a novel role and mechanism of Nox4 in regulating autophagy and ER-phagy during RANKL-induced osteoclastogenesis. Specifically, ER-localized Nox4 promotes RANKL-induced autophagy and ER-phagy by increasing ER-derived ROS and activating the UPR-related signaling pathway (PERK/elF-2a/ATF4). These findings may provide new insight into the processes of RANKL-induced osteoclastogenesis and help the development of new potential therapeutic strategies for osteoclastogenesis-related diseases. Future studies are needed to ascertain the functional role of Nox4related ER-phagy in osteoclastogenesis in vivo.

\section{Abbreviations}


RANKL: receptor activator of nuclear factor-KB ligand; CQ: chloroquine; Nox: NADPH oxidase; ER:

endoplasmic reticulum; ROS: reactive oxygen species; UPR: unfolded protein response; ER-phagy: endoplasmic reticulum autophagy; OCs: Osteoclasts; TRAP: tartrate-resistant acid phosphatase; PDB: Paget disease of bone; TNF: tumor necrosis factor; sRANKL: Recombinant murine soluble RANKL; DPI: Diphenyleneiodonium chloride; NAC: acetyl-L-cysteine; shRNA: short hairpin RNA; DMEM: Dulbecco's modified Eagle's medium; FBS: fetal bovine serum; MOI: multiplicity of infection; qRT-PCR: quantitative real-time PCR; TEM: Transmission electron microscopy; SDS-PAGE: sodium dodecyl sulfatepolyacrylamide; PVDF: polyvinylidene difluoride; HRP: horseradish peroxidase; ECL: chemiluminescence substrate; PBS: phosphate buffer saline. DCFH-DA: 2',7'-dichlorofluorescein diacetate; DCF: 2',7'dichlorofluorescein; ANOVA: one-way analysis of variance; ERQC: ER quality control system.

\section{Declaration}

\section{Acknowledgements}

All authors express great gratitude to Doctor Wei Liu for assistance in making graphs/figures and Doctor Min Ma for providing assistance in manuscript preparation.

\section{Authors' contributions}

JS have designed the research work, performed the experiments and drafted the manuscript. WC have performed experiments and substantively revised the manuscript; SL have performed experiments; SY made substantial contributions to data analysis; $Y Z$ have performed experiments and contributed to data interpretation; $\mathrm{XH}$ have planned experiments and performed experiments; $\mathrm{HQ}$ have performed experiments and revised the manuscript; $S X$ have have made substantial contributions to the conception of this research and the preparation of manuscript; TC have made substantial contributions to the conception and design of this work. All authors read and approved the final manuscript and approved its submission.

\section{Funding}

This work was funded by the National Natural Science Foundation of China (NSFC-81570800) and the National Natural Science Foundation Youth Project of China (No. 81501853). The content is solely the responsibility of the authors and does not necessarily represent the official views of the National Natural Science Foundation and the National Natural Science Foundation Youth Project. These funding agencies played no role in the design of the study; the collection, analysis, and interpretation of data; and the writing of the manuscript.

\section{Availability of data and materials}

Western blot datasets generated during the current study are available from the corresponding author on reasonable request. All other data generated or analyzed during this study are included in this published article and its supplementary information files. 


\section{Ethics approval and consent to participate}

Not applicable

\section{Consent for publication}

Not applicable

\section{Competing interests}

The authors declare that they have no competing interests.

\section{Reference}

1. Upadhyay J, Farr OM, Mantzoros CS. The role of leptin in regulating bone metabolism. METABOLISM. 2015; 64: 105-13; doi: 10.1016/j.metabol.2014.10.021.

2. Chung $Y H$, Jang $Y$, Choi B, Song DH, Lee EJ, Kim SM, et al. Beclin-1 is required for RANKL-induced osteoclast differentiation. J CELL PHYSIOL. 2014; 229: 1963-71; doi:10.1002/ jcp.24646.

3. Boyce BF. Advances in the regulation of osteoclasts and osteoclast functions. J DENT RES. 2013; 92 : 860-7; doi:10.1177/0022034513500306.

4. Bloemen V, Schoenmaker T, de Vries TJ, Everts V. Direct cell-cell contact between periodontal ligament fibroblasts and osteoclast precursors synergistically increases the expression of genes related to osteoclastogenesis. J CELL PHYSIOL. 2010; 222: 565-73; doi:10.1002/jcp.21971.

5. Chung PY, Van Hul W. Paget's disease of bone: evidence for complex pathogenetic interactions, Semin Arthritis Rheum. 2012; 41: 619-41; doi:10.1016/j.semarthrit.2011.07.005.

6. Johnson RW, Schipani E, Giaccia AJ. HIF targets in bone remodeling and metastatic disease. Pharmacol Ther. 2015; 150: 169-77; doi:10.1016/j.pharmthera.2015.02.002.

7. Boyce BF. Advances in osteoclast biology reveal potential new drug targets and new roles for osteoclasts. J BONE MINER RES. 2013; 28: 711-22; doi:10.1002/jbmr.1885.

8. Oikawa T, Kuroda Y, Matsuo K. Regulation of osteoclasts by membrane-derived lipid mediators. CELL MOL LIFE SCI. 2013; 70: 3341-53; doi:10.1007/s00018-012-1238-4

9. Pacifici R. Role of T cells in ovariectomy induced bone loss--revisited. J BONE MINER RES. 2012; 27: 231-9; doi:10.1002/jbmr.1500.

10. Kimura K, Kitaura H, Fujii T, Ishida M, Hakami ZW, Takano-Yamamoto T. An anti-c-Fms antibody inhibits osteoclastogenesis in a mouse periodontitis model. ORAL DIS. 2014; 20:319-24; doi:10.1111/odi.12117.

11. Feng X, Teitelbaum SL. Osteoclasts: New Insights. BONE RES. 2013; 1:11-26; doi:10.4248/ BR201301003.

12. Dahiya N, Khadka A, Sharma AK, Gupta AK, Singh N, Brashier DB. Denosumab: A bone antiresorptive drug, Med J Armed Forces India 2015; 71: 71-5; doi:10.1016/j.mjafi.2014.02.001. 
13. Kim KH, Lee MS. Autophagy--a key player in cellular and body metabolism. NAT REV ENDOCRINOL. 2014; 10: 322-37; doi:10.1038/nrendo.2014.35.

14. Singh R, Xiang $Y$, Wang $Y$, Baikati K, Cuervo AM, Luu YK, et al. Autophagy regulates adipose mass and differentiation in mice. J CLIN INVEST. 2009; 119: 3329-39; doi:10.1172/JCI39228.

15. Stappenbeck TS. The role of autophagy in Paneth cell differentiation and secretion. MUCOSAL IMMUNOL. 2010; 3: 8-10; doi:10.1038/mi.2009.12.

16. Zeng M, Zhou JN. Roles of autophagy and mTOR signaling in neuronal differentiation of mouse neuroblastoma cells. CELL SIGNAL. 2008; 20: 659-65; doi:10.1016/j.cellsig.2007.11.015.

17. Colosetti P, Puissant A, Robert G, Luciano F, Jacquel A, Gounon P, et al. Autophagy is an important event for megakaryocytic differentiation of the chronic myelogenous leukemia K562 cell line. AUTOPHAGY. 2009; 5: 1092-8; doi:10.4161/auto.5.8.9889.

18. De Meyer GR, Grootaert MO, Michiels CF, Kurdi A, Schrijvers DM, Martinet W. Autophagy in vascular disease, CIRC RES. 2015; 116: 468-79; doi:10.1161/CIRCRESAHA.116.303804.

19. Rogov V, Dotsch V, Johansen $T$, Kirkin V, Interactions between autophagy receptors and ubiquitin-like proteins form the molecular basis for selective autophagy. MOL CELL. 2014; 53: 167-78; doi:10.1016/j.molcel.2013.12.014.

20. Zhao Y, Chen G, Zhang W, Xu N, Zhu JY, Jia J, et al. Autophagy regulates hypoxia-induced osteoclastogenesis through the HIF-1alpha/BNIP3 signaling pathway. J CELL PHYSIOL. 2012; 227: 639-48; doi:10.1002/jcp.22768.

21. Shi J, Wang L, Zhang H, Jie Q, Li X, Shi Q, et al. Glucocorticoids: Dose-related effects on osteoclast formation and function via reactive oxygen species and autophagy. BONE. 2015; 79: 222-32; doi:10.1016/j.bone.2015.06.014.

22. Sambandam Y, Townsend MT, Pierce JJ, Lipman CM, Haque A, Bateman TA, et al. Microgravity control of autophagy modulates osteoclastogenesis. BONE. 2014; 61: 125-31; doi:10.1016/ j.bone.2014.01.004.

23. DeSelm CJ, Miller BC, Zou W, Beatty WL, van Meel E, Takahata Y, et al. Autophagy proteins regulate the secretory component of osteoclastic bone resorption. DEV CELL. 2011; 21: 966-74; doi:10.1016/j.devcel.2011.08.016.

24. Asai K, Funaba M, Murakami M. Enhancement of RANKL-induced MITF-E expression and osteoclastogenesis by TGF-beta. CELL BIOCHEM FUNCT. 2014; 32: 401-9; doi:10.1002/cbf.3028.

25. Sun KT, Chen MY, Tu MG, Wang IK, Chang SS, Li CY. MicroRNA-20a regulates autophagy related protein-ATG16L1 in hypoxia-induced osteoclast differentiation. BONE. 2015; 73: 145-53; doi:10.1016/j.bone.2014.11.026.

26. Wang K, Niu J, Kim H, Kolattukudy PE. Osteoclast precursor differentiation by MCPIP via oxidative stress, endoplasmic reticulum stress, and autophagy. J MOL CELL BIOL. 2011; 3: 360-8; doi:10.1093/jmcb/mjr021.

27. Sciarretta S, Zhai P, Shao D, Zablocki D, Nagarajan N, Terada LS, et al. Activation of NADPH oxidase 4 in the endoplasmic reticulum promotes cardiomyocyte autophagy and survival during energy 
stress through the protein kinase RNA-activated-like endoplasmic reticulum kinase/eukaryotic initiation factor 2alpha/activating transcription factor 4 pathway. CIRC RES. 2013; 113: 1253-64; doi:10.1161/CIRCRESAHA.113.301787.

28. Lee NK, Choi YG, Baik JY, Han SY, Jeong DW, Bae YS, et al. A crucial role for reactive oxygen species in RANKL-induced osteoclast differentiation. BLOOD. 2005; 106: 852-9; doi:10.1182/ blood-2004-093662.

29. Kang IS, Kim C. NADPH oxidase gp91(phox) contributes to RANKL-induced osteoclast differentiation by upregulating NFATc1. Sci Rep. 2016; 6: 38014; doi:10.1038/srep38014.

30. Goettsch C, Babelova A, Trummer O, Erben RG, Rauner M, Rammelt S, et al. NADPH oxidase 4 limits bone mass by promoting osteoclastogenesis. J CLIN INVEST. 2013; 123: 4731-8; doi:10.1172/ JCl67603.

31. Lassegue B, San MA, Griendling KK. Biochemistry, physiology, and pathophysiology of NADPH oxidases in the cardiovascular system. CIRC RES. 2012; 110: 1364-90; doi:10.1161/CIRCRESAHA. 111.243972.

32. Stephens SB, Nicchitta CV. Divergent regulation of protein synthesis in the cytosol and endoplasmic reticulum compartments of mammalian cells. MOL BIOL CELL. 2008; 19: 623-32; doi:10.1091/mbc. e07-07-0677.

33. Chen K, Kirber MT, Xiao H, Yang Y, Keaney JJ. Regulation of ROS signal transduction by NADPH oxidase 4 localization. J CELL BIOL. 2008; 181: 1129-39; doi:10.1083/jcb.200709049.

34. Finkel T. Signal transduction by reactive oxygen species, J CELL BIOL 2011; 194: 7-15; doi:10.1083/jcb.201102095.

35. Kongara S, Karantza V. The interplay between autophagy and ROS in tumorigenesis. FRONT ONCOL. 2012; 2: 171; doi:10.3389/fonc.2012.00171.

36. Avivar-Valderas A, Salas E, Bobrovnikova-Marjon E, Diehl JA, Nagi C, Debnath J, et al. PERK integrates autophagy and oxidative stress responses to promote survival during extracellular matrix detachment. MOL CELL BIOL. 2011; 31: 3616-29; doi:10.1128/MCB.05164-11.

37. Walter P, Ron D. The unfolded protein response: from stress pathway to homeostatic regulation. SCIENCE. 2011; 334: 1081-6; doi:10.1126/science.1209038.

38. Jiang S, Lin Y, Yao H, Yang C, Zhang L, Luo B, et al. The role of unfolded protein response and ERphagy in quantum dots-induced nephrotoxicity: an in vitro and in vivo study. ARCH TOXICOL. 2018; 92: 1421-34; doi:10.1007/s00204-018-2169-0.

39. Shapiro IM, Layfield R, Lotz M, Settembre C, Whitehouse C, Boning up on autophagy: the role of autophagy in skeletal biology. AUTOPHAGY. 2014; 10: 7-19; doi:10.4161/auto.26679.

40. Kleniewska P, Piechota A, Skibska B, Goraca A. The NADPH oxidase family and its inhibitors. Arch Immunol Ther Exp (Warsz). 2012; 60: 277-94; doi:10.1007/s00005-012-0176-z.

41. Youn JY, Zhang J, Zhang Y, Chen H, Liu D, Ping P, et al. Oxidative stress in atrial fibrillation: an emerging role of NADPH oxidase. J MOL CELL CARDIOL. 2013; 62: 72-9; doi:10.1016/j.yjmcc. 2013.04.019. 
42. Sasaki H, Yamamoto H, Tominaga K, Masuda K, Kawai T, Teshima-Kondo S, et al. Receptor activator of nuclear factor-kappaB ligand-induced mouse osteoclast differentiation is associated with switching between NADPH oxidase homologues. Free Radic Biol Med. 2009; 47: 189-99; doi:10.1016/j.freeradbiomed.2009.04.025.

43. Panday A, Sahoo MK, Osorio D, Batra S. NADPH oxidases: an overview from structure to innate immunity-associated pathologies. CELL MOL IMMUNOL. 2015; 12: 5-23; doi:10.1038/cmi.2014.89.

44. Zhang M, Perino A, Ghigo A, Hirsch E, Shah AM. NADPH oxidases in heart failure: poachers or gamekeepers? Antioxid Redox Signal. 2013; 18: 1024-41; doi:10.1089/ars.2012.4550.

45. Auer S, Rinnerthaler M, Bischof J, Streubel MK, Breitenbach-Koller H, Geisberger R, et al. The Human NADPH Oxidase, Nox4, Regulates Cytoskeletal Organization in Two Cancer Cell Lines, HepG2 and SH-SY5Y. FRONT ONCOL. 2017; 7: 111; doi:10.3389/fonc.2017.00111.

46. Ago T, Kuroda J, Pain J, Fu C, Li H, Sadoshima J. Upregulation of Nox4 by hypertrophic stimuli promotes apoptosis and mitochondrial dysfunction in cardiac myocytes. CIRC RES. 2010; 106: 125364; doi:10.1161/CIRCRESAHA.109.213116.

47. Wu RF, Ma Z, Liu Z, Terada LS. Nox4-derived H2O2 mediates endoplasmic reticulum signaling through local Ras activation. MOL CELL BIOL. 2010; 30: 3553-68; doi:10.1128/MCB.01445-09.

48. Anilkumar N, San JG, Sawyer I, Santos CX, Sand C, Brewer AC, et al. A 28-kDa splice variant of NADPH oxidase-4 is nuclear-localized and involved in redox signaling in vascular cells. Arterioscler Thromb Vasc Biol. 2013; 33: e104-12; doi:10.1161/ATVBAHA.112.300956.

49. Colombo S, Longhi R, Alcaro S, Ortuso F, Sprocati T, Flora A, et al. N-myristoylation determines dual targeting of mammalian NADH-cytochrome b5 reductase to ER and mitochondrial outer membranes by a mechanism of kinetic partitioning. J CELL BIOL. 2005; 168: 735-45; doi:10.1083/ jcb.200407082.

50. Canugovi C, Stevenson MD, Vendrov AE, Hayami T, Robidoux J, Xiao H, et al. Increased mitochondrial NADPH oxidase 4 (NOX4) expression in aging is a causative factor in aortic stiffening. REDOX BIOL. 2019; 26: 101288; doi:10.1016/j.redox.2019.101288.

51. Scherz-Shouval R, Elazar Z. Regulation of autophagy by ROS: physiology and pathology. TRENDS BIOCHEM SCI. 2011; 36: 30-8; doi:10.1016/j.tibs.2010.07.007.

52. Lee J, Giordano S, Zhang J. Autophagy, mitochondria and oxidative stress: cross-talk and redox signalling. BIOCHEM J. 2012; 441: 523-40; doi:10.1042/BJ2011145

53. Kim JY, Kim JK, Kim H. ABCB7 simultaneously regulates apoptotic and non-apoptotic cell death by modulating mitochondrial ROS and HIF1alpha-driven NFkappaB signaling. ONCOGENE. 2019; doi:10.1038/s41388-019-1118-6.

54. Srinivasan S, Koenigstein A, Joseph J, Sun L, Kalyanaraman B, Zaidi M, et al. Role of mitochondrial reactive oxygen species in osteoclast differentiation. Ann N Y Acad Sci. 2010; 1192: 245-52; doi:10.1111/j.1749-6632.2009.05377.x

55. Fregno I, Molinari M. Endoplasmic reticulum turnover: ER-phagy and other flavors in selective and non-selective ER clearance. F1000Res. 2018; 7: 454; doi:10.12688/f1000research.13968.1. 
56. Lipatova Z, Segev N. A Role for Macro-ER-Phagy in ER Quality Control. PLOS GENET. 2015; 11: e1005390; doi:10.1371/journal.pgen.1005390.

57. Mochida K, Oikawa Y, Kimura Y, Kirisako H, Hirano H, Ohsumi Y, et al. Receptor-mediated selective autophagy degrades the endoplasmic reticulum and the nucleus. NATURE. 2015; 522: 359-62; doi:10.1038/nature14506.

58. Khaminets A, Heinrich T, Mari M, Grumati P, Huebner AK, Akutsu M, et al. Regulation of endoplasmic reticulum turnover by selective autophagy. NATURE. 2015; 522: 354-8; doi:10.1038/ nature14498.

59. Song S, Tan J, Miao Y, Zhang Q. Crosstalk of ER stress-mediated autophagy and ER-phagy: Involvement of UPR and the core autophagy machinery. J CELL PHYSIOL. 2018; 233: 3867-74; doi:10.1002/jcp.26137.

\section{Figures}


A

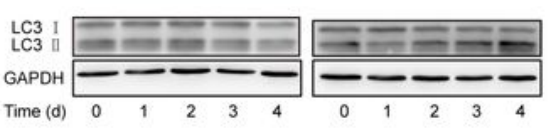

$\begin{array}{lllllllllll}\text { RANKL (ng/mL) } & 0 & 0 & 0 & 0 & 0 & 100 & 100 & 100 & 100 & 100\end{array}$
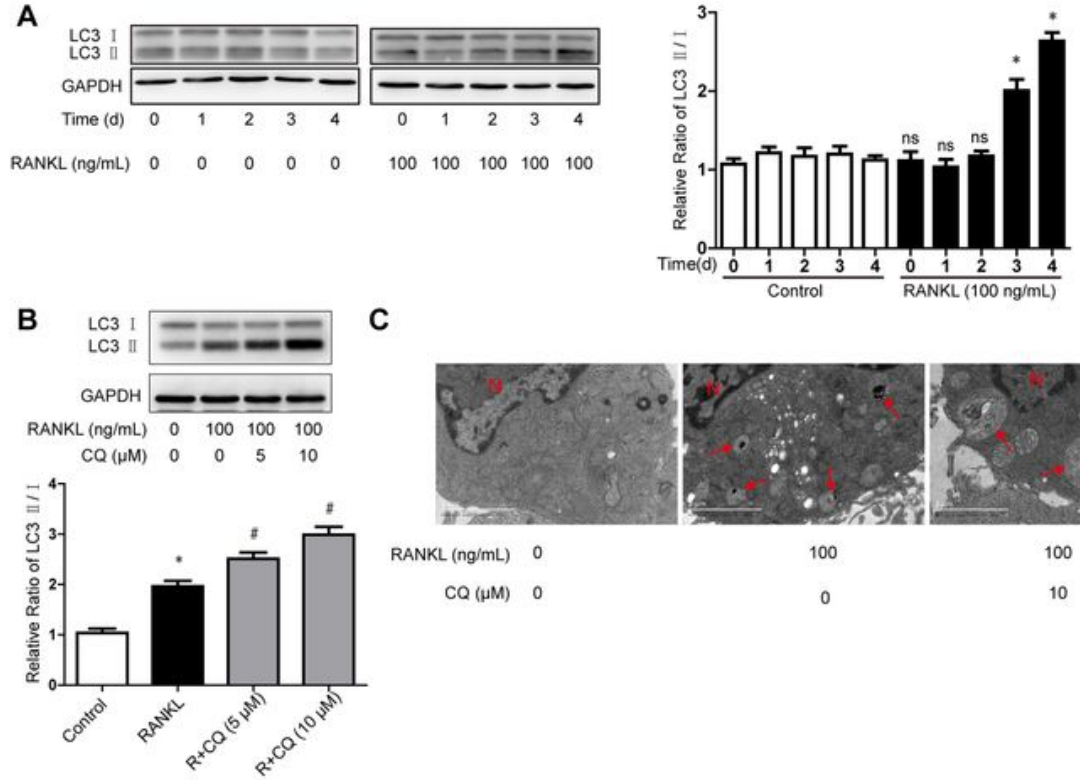

C

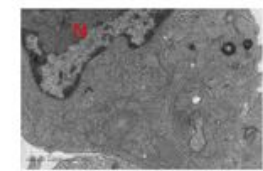

RANKL (ng/mL) 0

$\mathrm{CQ}(\mu \mathrm{M}) \quad 0$

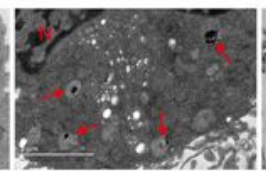

100
100
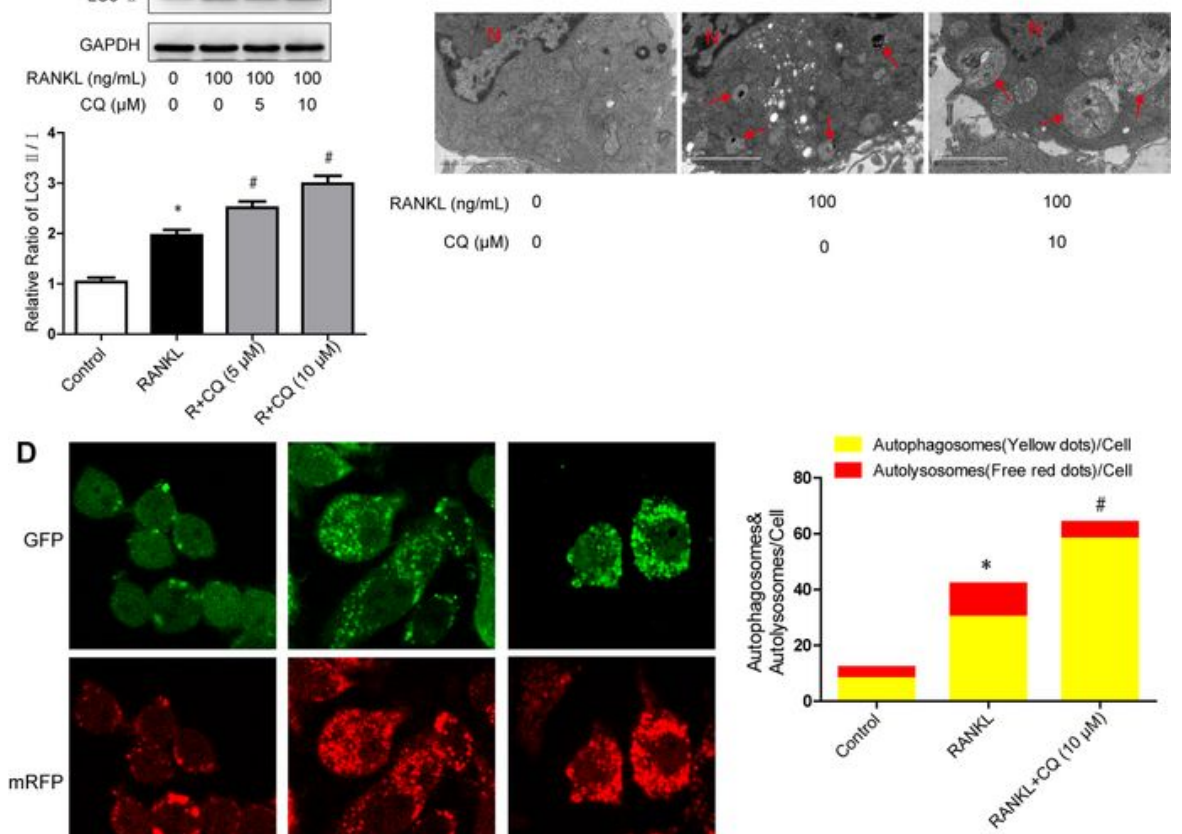

mRFP
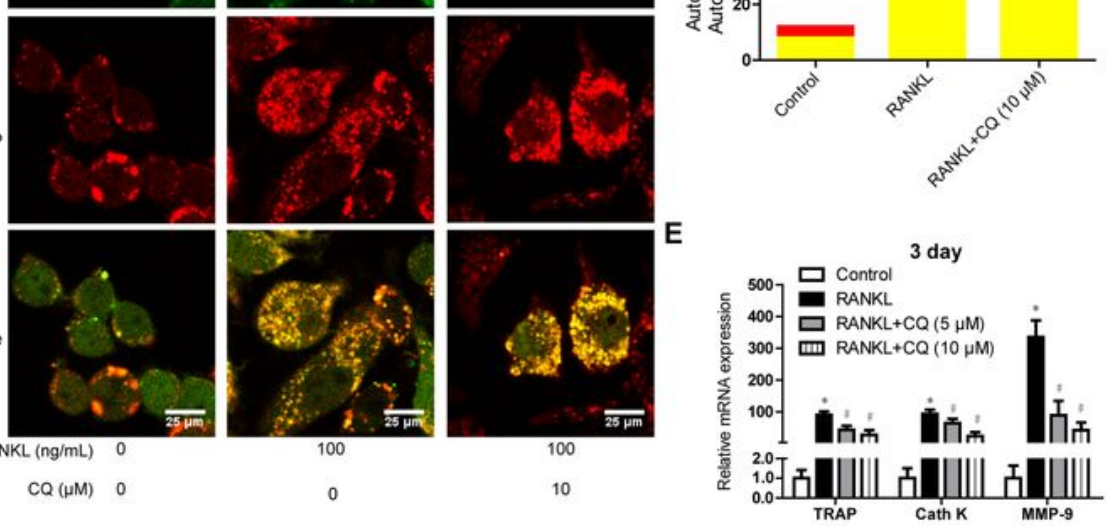

$\mathbf{F}$

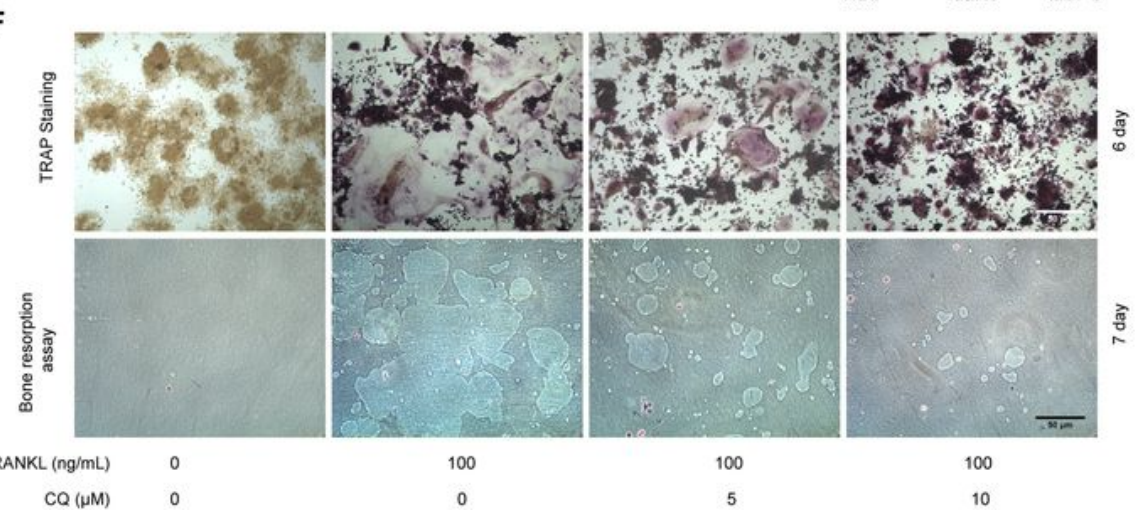

Figure 1

RANKL induces osteoclastogenesis and bone resorption via autophagy. (A) RAW264.7 cells were seeded overnight and incubated with or without RANKL $(100 \mathrm{ng} / \mathrm{mL})$ for the indicated times (0-4 days). The protein levels of LC3-I and LC3-II were tested by western blot, and the ratio of LC3-II/LC3-I was quantified by Image J. (B) RAW264.7 cells were seeded overnight and treated with the autophagy inhibitor CQ (5 and $10 \mu \mathrm{M})$ in the presence of RANKL $(100 \mathrm{ng} / \mathrm{mL})$ for 3 days. The ratio of LC3-II/LC3-I was detected and 
quantified as described in (A). (C) RAW264.7 cells were seeded overnight and treated with CQ $(10 \mu \mathrm{M})$ in the presence of RANKL $(100 \mathrm{ng} / \mathrm{mL})$ for 3 days. The autophagic vacuoles (autophagosomes and autolysosomes) were monitored by TEM. Representative TEM images are shown, and the typical autophagic vacuoles are marked with red arrows. (D) After transfection with Ad-mRFP-GFP-LC3 for $48 \mathrm{~h}$, RAW264.7 cells were treated as described in (C). Then, autophagic flux was assessed by quantifying the number of mRFP and GFP puncta per cell under a laser scanning confocal microscope. Representative images of mRFP and GFP puncta are shown, together with the quantification of autophagosomes and autolysosomes. (E) RAW264.7 cells were treated as described in (B). Then, the mRNA expression levels of osteoclastogenesis-related genes (TRAP, Cath K and MMP-9) were detected by qRT-PCR. (F) RAW264.7 cells were treated as described in (B) for the indicated times ( 6 and 7 days). Then, TRAP staining and bone resorption assays were performed to evaluate the formation of TRAP-positive multinucleated $(\geq 3)$ osteoclasts and bone resorption pits, respectively, under a light microscope. All the data are presented as the mean \pm SD from three independent experiments. ns, no significance; ${ }^{*} P<0.05$ versus the control group; $\# \mathrm{P}<0.05$ versus the RANKL group. 
A

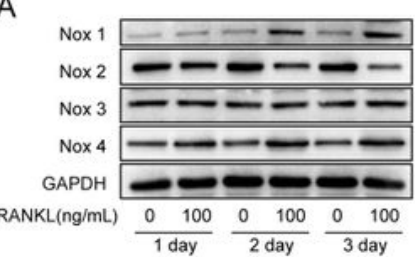

C

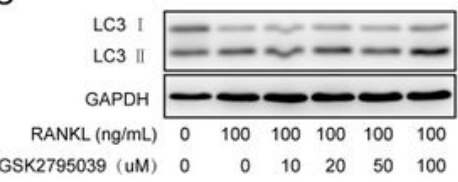

B
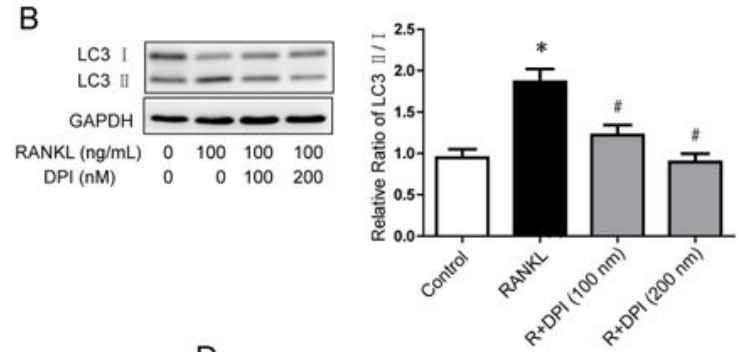

D
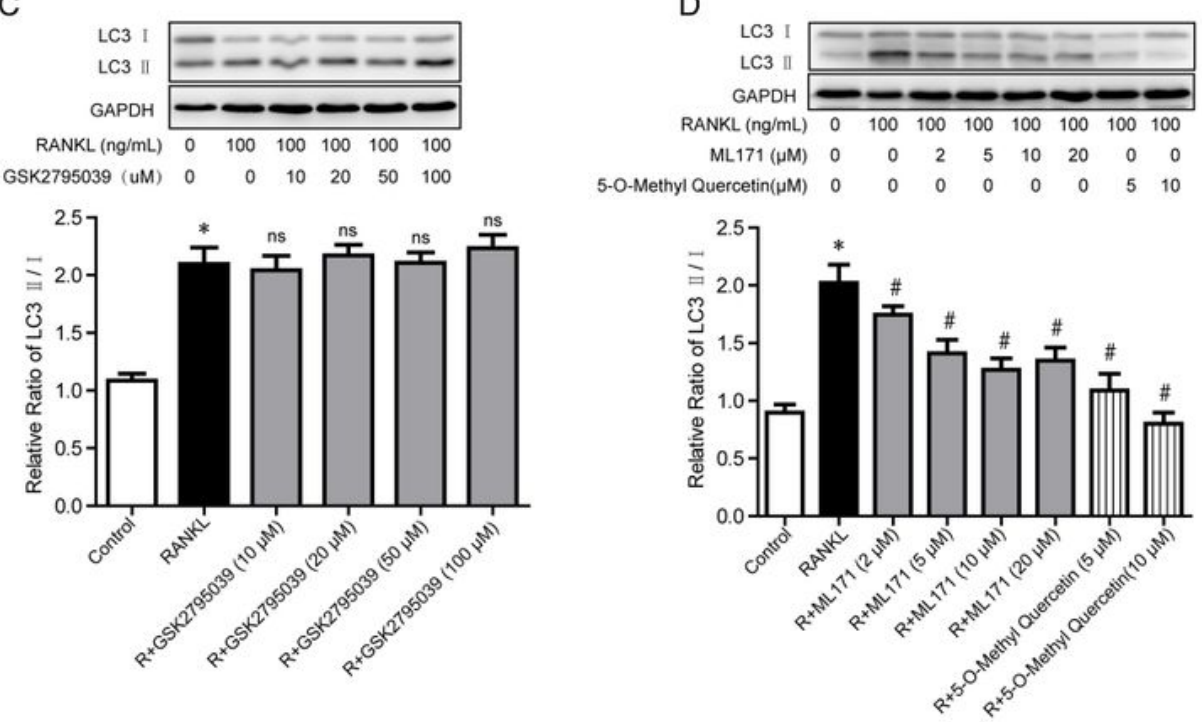

$\mathrm{E}$

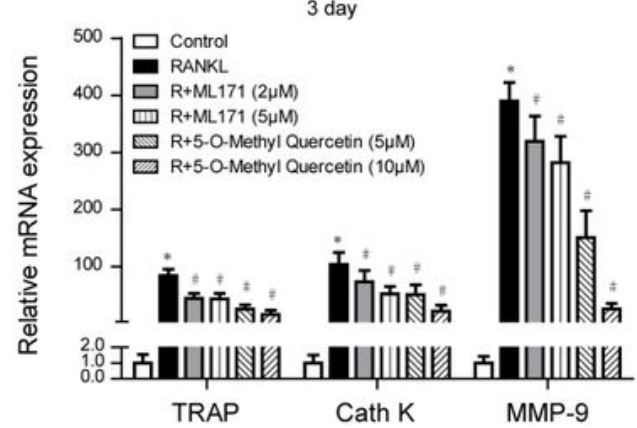

$\mathrm{F}$
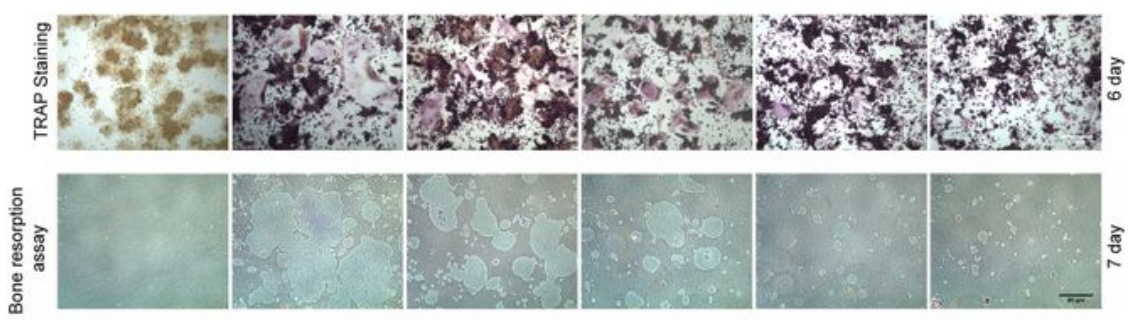

RANKL (ng/mL) 0 ML171 $(\mu \mathrm{M}) \quad 0$ 5-O-Methyl Quercetin( $\mu \mathrm{M}) \quad 0$

100
0

100

2
0

100

5
0

100

100

0

\section{Figure 2}

The inhibition of Nox4 suppresses RANKL-induced autophagy and osteoclastogenesis. (A) RAW264.7 cells were seeded overnight and incubated with or without RANKL $(100 \mathrm{ng} / \mathrm{mL})$ for the indicated times (13 days). The levels of Nox family proteins (Nox1, Nox2, Nox3 and Nox4) were assessed by western blot. (B-D) RAW264.7 cells were seeded overnight and treated with the Nox inhibitor DPI (B), Nox2 inhibitor GSK2795039 (C), Nox1 inhibitor ML171 and Nox4 inhibitor 5-0-methyl quercetin (D) in the presence of 
RANKL (100 ng/mL) for 3 days. The protein levels of LC3-I and LC3-II were tested by western blot, and the ratio of LC3-II/LC3-I was quantified by Image J. (E) RAW264.7 cells were seeded overnight and incubated with ML171 (2 and $5 \mu \mathrm{M})$ and 5-0-methyl quercetin (5 and $10 \mu \mathrm{M})$ in the presence of RANKL $(100 \mathrm{ng} / \mathrm{mL})$ for 3 days. The mRNA expression levels of TRAP, Cath $K$ and MMP-9 were detected by qRT-PCR. (F) RAW264.7 cells were treated as described in (E) for the indicated times ( 6 and 7 days). Then, TRAP staining and bone resorption assays were performed to evaluate the formation of TRAP-positive multinucleated $(\geq 3)$ osteoclasts and bone resorption pits, respectively, under a light microscope. All the data are presented as the mean \pm SD from three independent experiments. ${ }^{*}<0.05$ versus the control group; ns, no significance; \#P $<0.05$ versus the RANKL group. 
A

Nox4

VDAC1

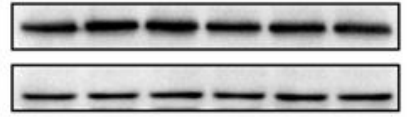

$\operatorname{RANKL}(\mathrm{ng} / \mathrm{mL}) \frac{0100}{1 \text { day }} \frac{0100}{2 \text { day }} \frac{0100}{3 \text { day }}$

D

ER-Tracker

DAPI
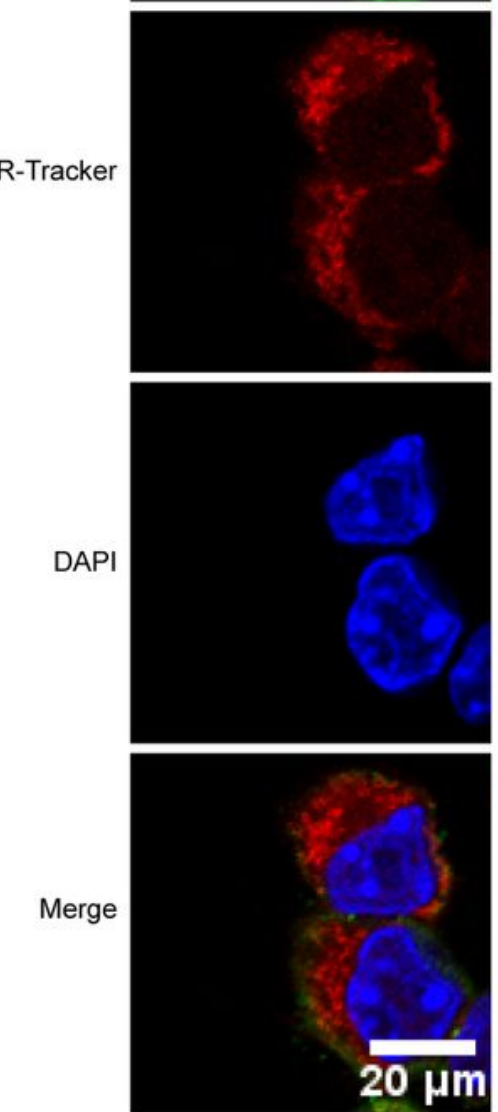

$\operatorname{RANKL}(\mathrm{ng} / \mathrm{mL})$

0

sh-NC

sh-Nox4
B

Nox4

ERp57

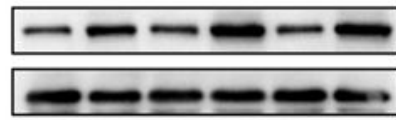

$\operatorname{RANKL}(\mathrm{ng} / \mathrm{mL}) \frac{0100}{1 \text { day }} \quad \frac{0100}{2 \text { day }} \quad \frac{0100}{3 \text { day }}$
C

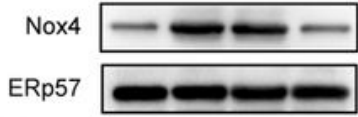

RANKL (ng/mL) $0 \quad 100 \quad 100 \quad 100$

sh-NC

sh-Nox4
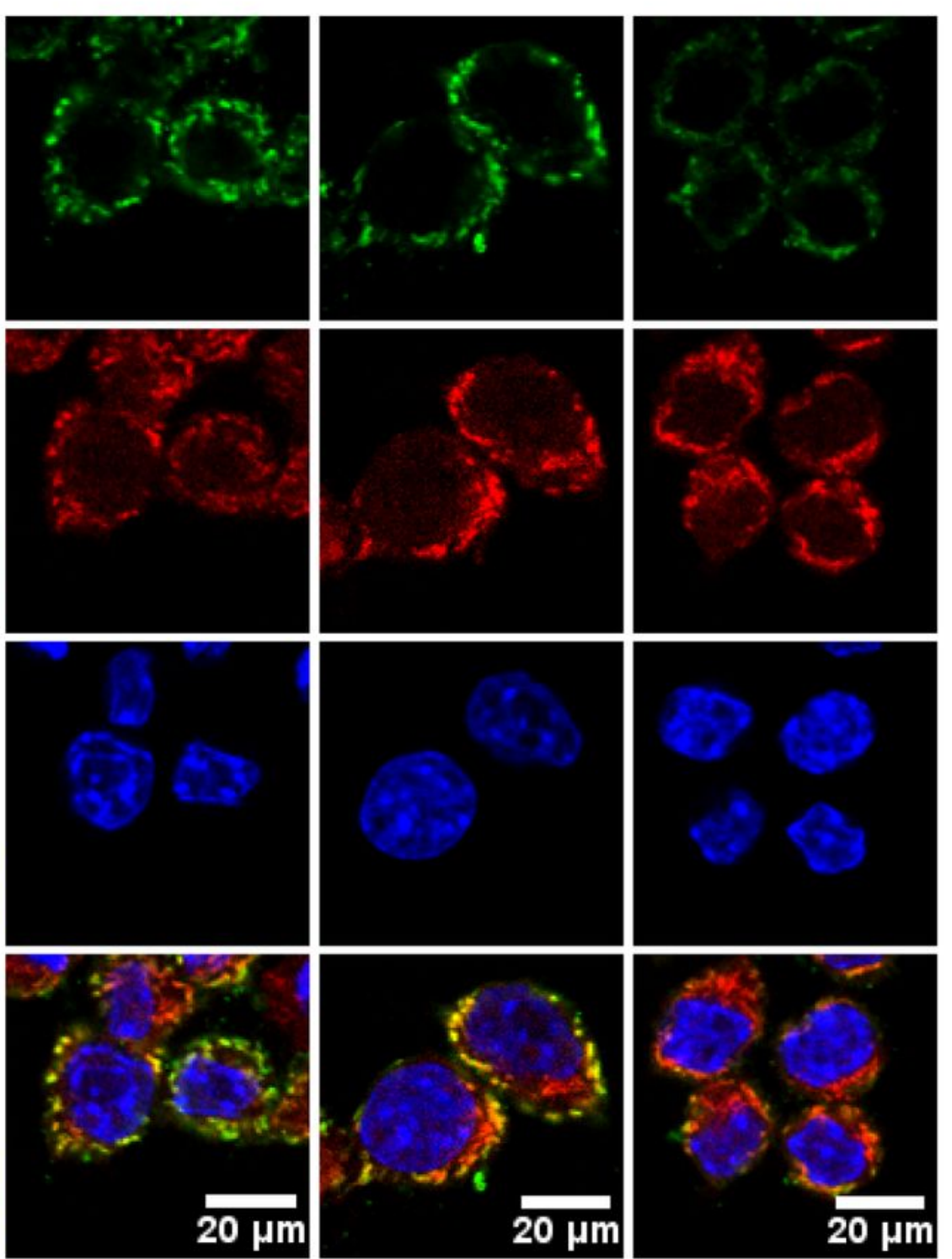

100

100

100

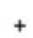

\section{Figure 3}

RANKL treatment specifically upregulates the level of Nox4 protein in the ER. (A-B) RAW264.7 cells were seeded overnight and incubated with or without RANKL $(100 \mathrm{ng} / \mathrm{mL})$ for the indicated times (1-3 days). The mitochondrial (A) and ER (B) fractions were isolated from cells. The protein levels of Nox4 in each fraction were assessed by western blot. VDAC1 and ERp57 were separately used as loading controls for mitochondria and the ER. (C) After sh-NC or sh-Nox4 transfection, RAW264.7 cells were seeded overnight 
and incubated with or without RANKL $(100 \mathrm{ng} / \mathrm{mL})$ for 3 days. The ER fraction was isolated from cells. The protein level of Nox4 in the ER was assessed by western blot. (D) RAW264.7 cells were treated as described in (C). Then, the cells were stained with ER-Tracker ${ }^{\text {TM }}$ Blue-White DPX. Immunofluorescence staining of Nox4 was performed to detect the colocalization of Nox4 with the ER.
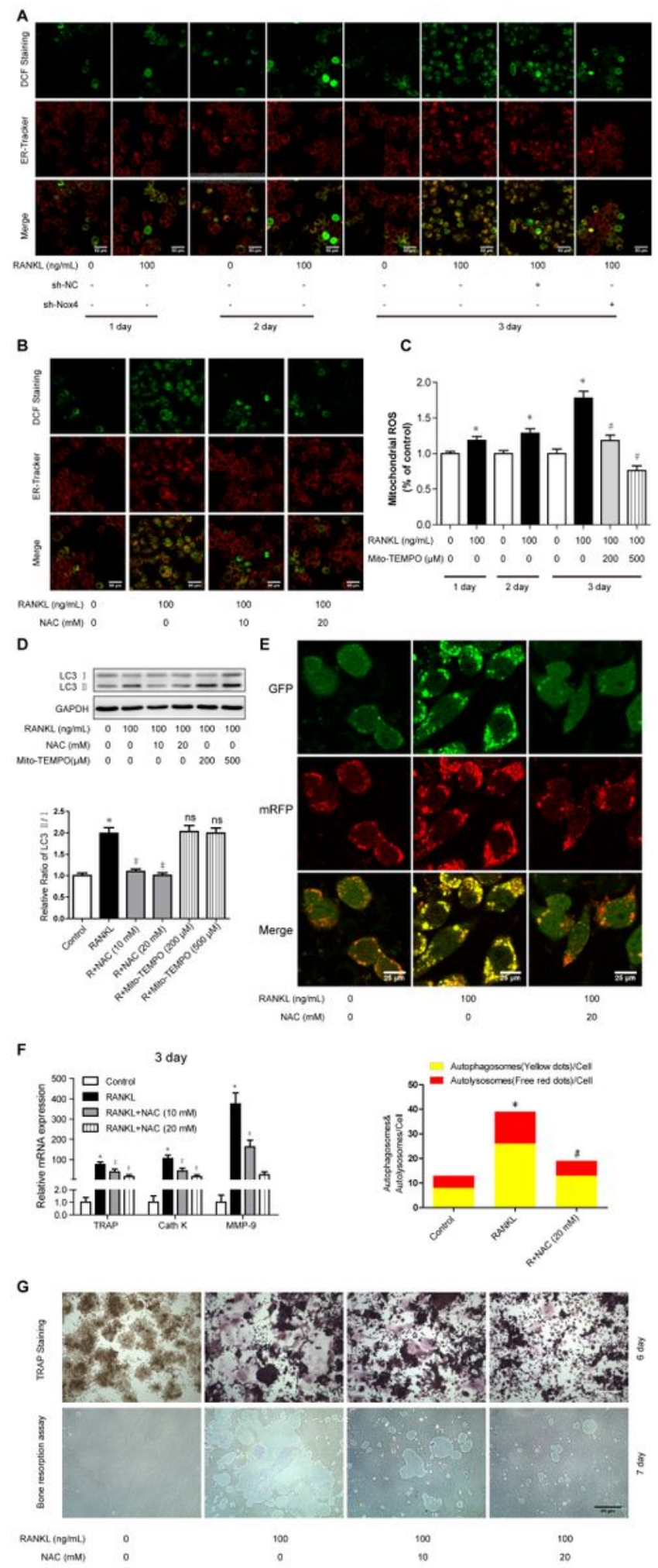

Figure 4 
Nox4 promotes RANKL-induced autophagy and osteoclastogenesis via generating nonmitochondrial ROS. (A) After sh-NC or sh-Nox4 transfection, RAW264.7 cells were cultured with or without RANKL (100 $\mathrm{ng} / \mathrm{mL}$ ) for the indicated times (1-3 days). Then, the cells were stained with ER-Tracker ${ }^{\mathrm{TM}}$ Blue-White DPX. DCF fluorescence staining was performed to evaluate the level of intracellular ROS. (B) RAW264.7 cells were treated with the intracellular ROS scavenger NAC (10 and $20 \mathrm{mM})$ in the presence of RANKL (100 $\mathrm{ng} / \mathrm{mL}$ ) for 3 days. The intracellular ROS and ER ROS were measured as described in (A). (C) RAW264.7 cells were treated with the mitochondrial-targeted antioxidant MitoTEMPO (200 and $500 \mu \mathrm{M})$ in the presence of RANKL $(100 \mathrm{ng} / \mathrm{mL})$. The level of mitochondrial ROS was measured by MitoSOX. (D) RAW264.7 cells were treated with NAC (10 and $20 \mathrm{mM})$ or MitoTEMPO $(200$ and $500 \mu \mathrm{M})$ in the presence of RANKL $(100 \mathrm{ng} / \mathrm{mL})$ for 3 days. Protein levels of LC3-I and LC3-II were tested by western blot, and the ratio of LC3-II/LC3-I was quantified by Image J. (E) After transfection with Ad-mRFP-GFP-LC3 for $48 \mathrm{~h}$, RAW264.7 cells were treated with NAC $(20 \mathrm{mM})$ in the presence of RANKL $(100 \mathrm{ng} / \mathrm{mL})$ for 3 days. Representative images of mRFP and GFP puncta are shown, together with quantification of autophagosomes and autolysosomes. (F) RAW264.7 cells were treated as described in (B) Then, the mRNA expression levels of TRAP, Cath K and MMP-9 were detected by qRT-PCR. (G) RAW264.7 cells were treated as described in (B) for the indicated times ( 6 and 7 days). TRAP-positive multinucleated $(\geq 3)$ osteoclasts and bone resorption pits were evaluated by TRAP staining and bone resorption assays, respectively. All data are from at least three independent experiments. ${ }^{*} \mathrm{P}<0.05$ versus the control group; $\# P<0.05$ versus the RANKL group. 

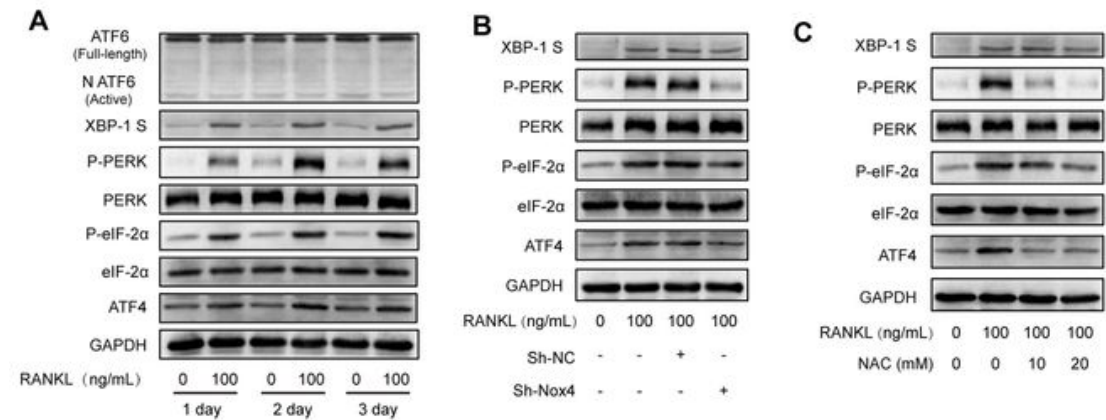

RANKL (ng/mL) $0 \quad 100 \quad 100 \quad 100$

NAC (mM) $000010 \quad 20$
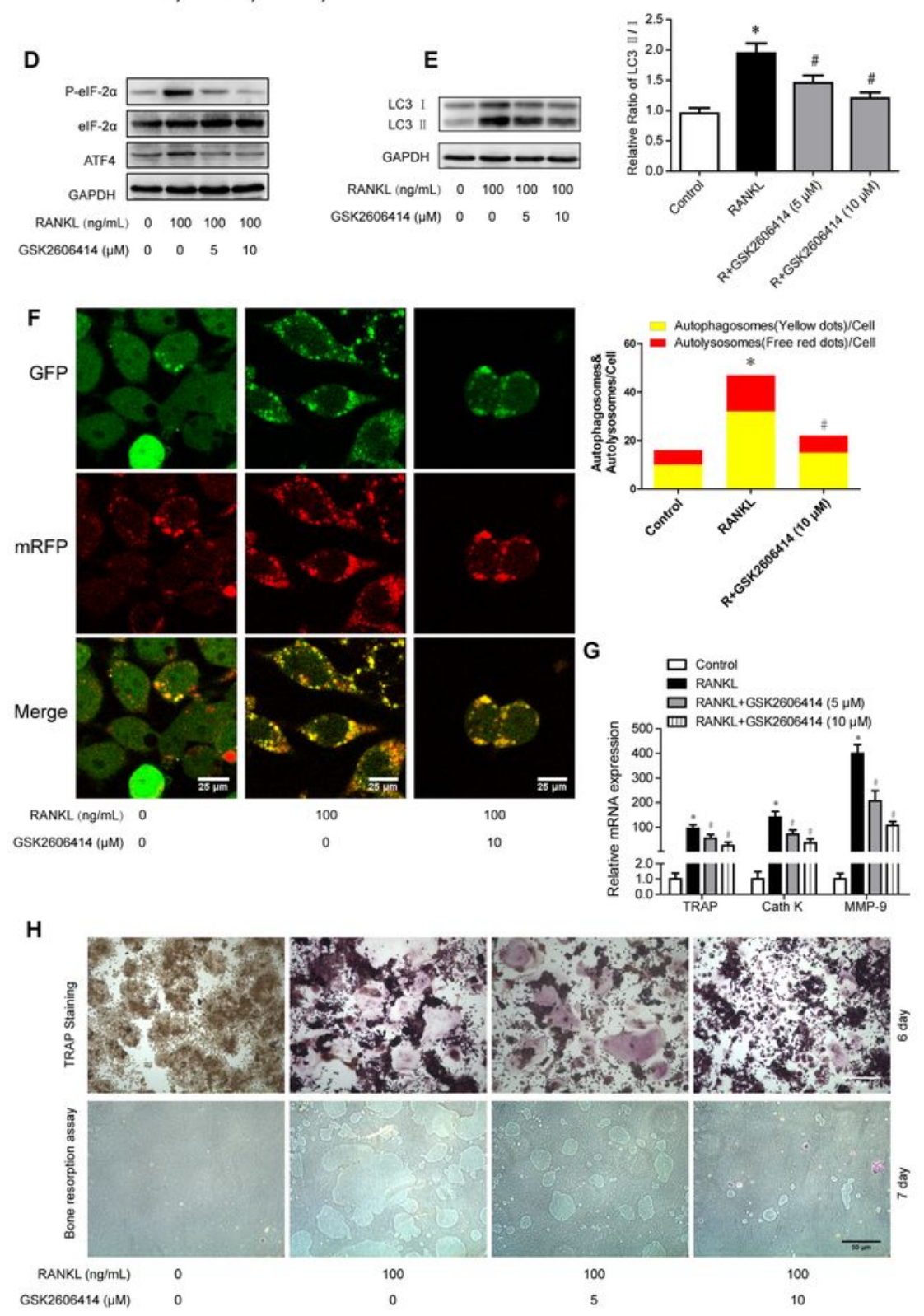

\section{Figure 5}

Nox4-derived ROS promotes autophagy via the PERK/elF-2a/ATF4 pathway. (A) RAW264.7 cells cultured with or without RANKL $(100 \mathrm{ng} / \mathrm{mL})$ for the indicated times. The protein levels of ATF6, XBP-1S, phosphoPERK (Thr980), PERK, phospho-elF-2 $a$ (Ser51), elF-2 $a$ and ATF4 were evaluated by western blot. (B) After sh-NC or sh-Nox4 transfection, RAW264.7 cells were incubated with or without RANKL $(100 \mathrm{ng} / \mathrm{mL})$ for 3 days. Protein levels were assessed by western blot. (C) RAW264.7 cells were treated with NAC (10 and 20 
$\mathrm{mM})$ and RANKL $(100 \mathrm{ng} / \mathrm{mL})$ for 3 days. The proteins mentioned in (A) were assessed by western blot. (D) RAW264.7 cells were incubated with PERK inhibitor GSK2606414 (5 and $10 \mu \mathrm{M})$ in the presence of RANKL ( $100 \mathrm{ng} / \mathrm{mL}$ ) for 3 days. The protein levels of phospho-elF-2a (Ser51), elF-2 $a$ and ATF4 were evaluated by western blot. (E) RAW264.7 cells were treated as described in (D). Then, the protein levels of LC3-I and LC3-II were tested by western blot, and the ratio of LC3-II/LC3-I was quantified by Image J. (F) After transfection with Ad-mRFP-GFP-LC3 for 48 h, RAW264.7 cells were treated with GSK2606414 (10 $\mu \mathrm{M})$ in the presence of RANKL $(100 \mathrm{ng} / \mathrm{mL})$ for 3 days. Autophagic flux was assessed by quantifying the mRFP and GFP puncta per cell under a laser scanning confocal microscope. Representative images of mRFP and GFP puncta are shown, together with quantification of autophagosomes and autolysosomes. (G) RAW264.7 cells were treated as in (D). Then, the mRNA expression levels of TRAP, Cath K and MMP-9 were detected by qRT-PCR. (H) RAW264.7 cells were treated as in (D) for indicated times ( 6 and 7 days). TRAP-positive multinucleated $(\geq 3$ ) osteoclasts and bone resorption pits were evaluated by TRAP staining and bone resorption assays, respectively. ${ }^{*} \mathrm{P}<0.05$ versus the control group; $\# \mathrm{P}<0.05$ versus the RANKL group. 


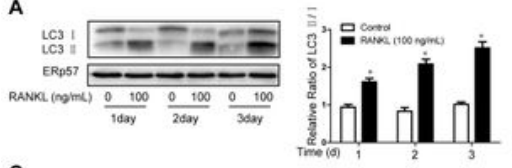

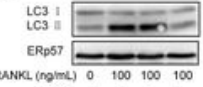

$\operatorname{sh} N$ NC
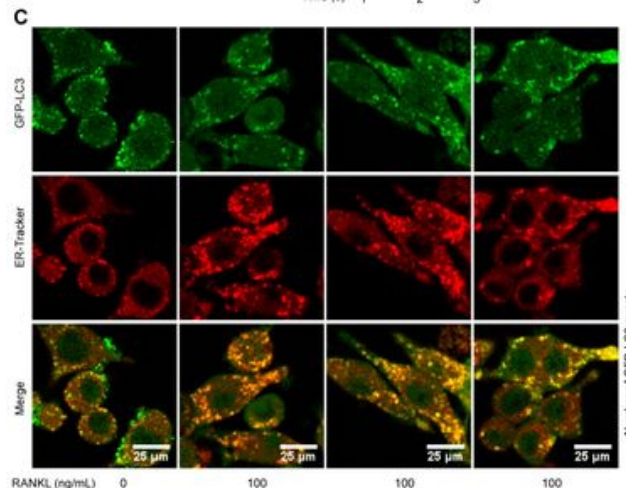

,

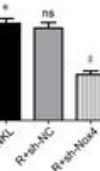

shinC

D

E
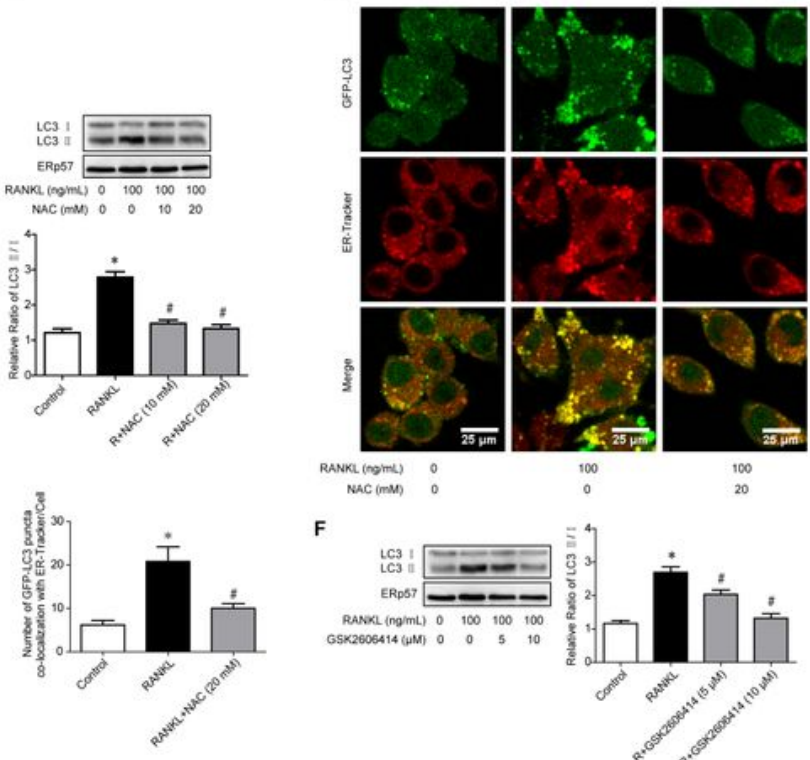

F
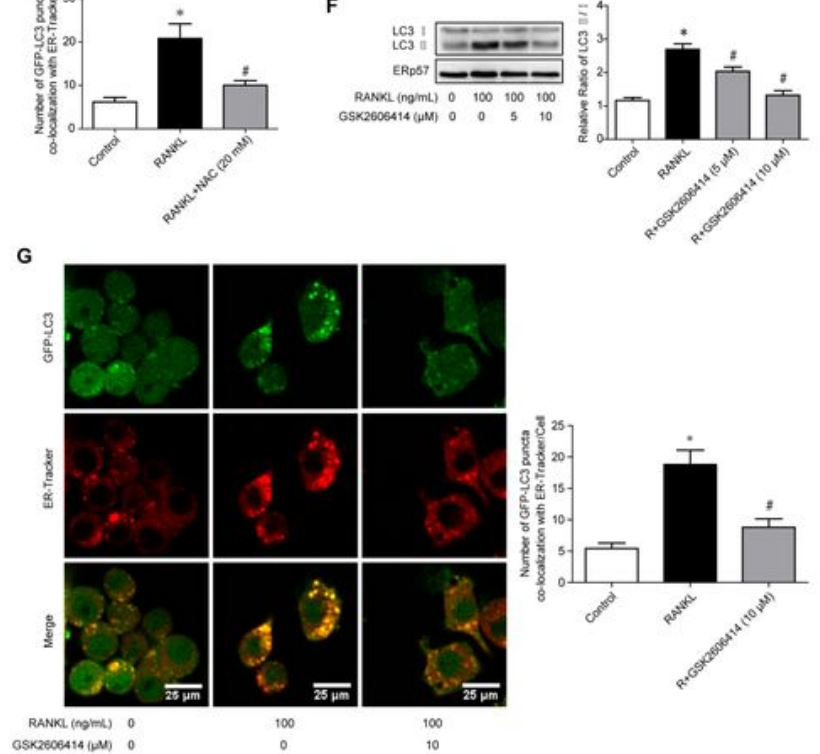

Figure 6

RANKL promotes ER-phagy by activating the Nox4/ROS/PERK/elF-2a/ATF4 pathway. (A) RAW264.7 cells were cultured with or without RANKL $(100 \mathrm{ng} / \mathrm{mL})$ for the indicated times (1-3 days). The protein levels of LC3-I and LC3-II in the ER were tested by western blot, and the ratio of LC3-II/LC3-I was quantified by Image J. ERp57 was used as a loading control. (B) After transfection with sh-NC or sh-Nox4, RAW264.7 cells were treated with or without RANKL $(100 \mathrm{ng} / \mathrm{mL})$ for 3 days. The ratio of LC3-II/LC3-I in the ER was 
detected and quantified as described in (A). (C) Representative images of GFP-LC3 puncta and ER-Tracker fluorescence. After transfection with sh-NC or sh-Nox4, RAW264.7 cells were transfected with Ad-GFP-LC3 for $48 \mathrm{~h}$. Then, the cells were treated with or without RANKL $(100 \mathrm{ng} / \mathrm{mL})$ for 3 days. GFP-LC3 puncta colocalization with ER-Tracker were quantified for comparison. (D) RAW264.7 cells were treated with NAC (10 and $20 \mathrm{mM})$ in the presence of RANKL $(100 \mathrm{ng} / \mathrm{mL})$ for 3 days. The ratio of LC3-II/LC3-I in the ER was detected and quantified as described in (A). (E) RAW264.7 cells were treated with NAC (20 mM) in the presence of RANKL (100 ng/mL) for 3 days. The colocalization of GFP-LC3 puncta with ER-Tracker was determined as described in (C). (F) RAW264.7 cells were treated with GSK2606414 (5 and $10 \mu \mathrm{M})$ in the presence of RANKL $(100 \mathrm{ng} / \mathrm{mL})$ for 3 days. The ratio of LC3-II/LC3-I in the ER was detected and quantified as described in (A). (G) RAW264.7 cells were treated with GSK2606414 (10 $\mu \mathrm{M})$ in the presence of RANKL $(100 \mathrm{ng} / \mathrm{mL})$ for 3 days. Colocalization of GFP-LC3 puncta with ER-Tracker was determined as described in (C). All data derived from three independent experiments. ${ }^{*} \mathrm{P}<0.05$ versus the control group; ns, no significance; \#P $<0.05$ versus the RANKL group.

\section{Supplementary Files}

This is a list of supplementary files associated with this preprint. Click to download.

- RawMaterialofwesternblot.rar

- SupplementalFig.1.tif

- SupplementalFig.2.tif 